\title{
MULTI-SCALE S-FRACTION REDUCED-ORDER MODELS FOR MASSIVE WAVEFIELD SIMULATIONS
}

\author{
VLADIMIR DRUSKIN*, ALEXANDER V. MAMONOV ${ }^{\dagger}$, AND MIKHAIL ZASLAVSKY*
}

\begin{abstract}
We developed a novel reduced-order multi-scale method for solving large timedomain wavefield simulation problems. Our algorithm consists of two main stages. During the first "off-line" stage the fine-grid operator (of the graph Laplacian type is partitioned on coarse cells (subdomains). Then projection-type multi-scale reduced order models (ROMs) are computed for the coarse cell operators. The off-line stage is embarrassingly parallel as ROM computations for the subdomains are independent of each other. It also does not depend on the number of simulated sources (inputs) and it is performed just once before the entire time-domain simulation. At the second "on-line" stage the time-domain simulation is performed within the obtained multi-scale ROM framework. The crucial feature of our formulation is the representation of the ROMs in terms of matrix Stieltjes continued fractions (S-fractions). The layered structure of the S-fraction introduces several hidden layers in the ROM representation, that results in the block-tridiagonal dynamic system within each coarse cell. This allows us to sparsify the obtained multi-scale subdomain operator ROMs and to reduce the communications between the adjacent subdomains which is highly beneficial for a parallel implementation of the on-line stage. Our approach suits perfectly the high performance computing architectures, however in this paper we present rather promising numerical results for a serial computing implementation only. These results include $3 D$ acoustic and multi-phase anisotropic elastic problems.
\end{abstract}

1. Introduction. Our main goal is to speed up the simulations of large-scale time-domain wave propagation problems using model order reduction techniques. To construct reduced order models (ROMs) we need a reference full-scale model. Such a model comes from a spatial discretization of a second order hyperbolic PDE (or a system of PDEs) on a reference fine grid, e.g., the scalar wave equation, linear elastodynamic system or Maxwell equations in wave regime.

Specifically, we consider the impulse source problem for a semi-discrete (discrete in space, continuous in time) system of linear second-order equations

$$
\mathbf{A} u-\mathbf{B} u_{t t}=g \delta(t),\left.\quad u\right|_{t<0}=0 .
$$

where $0 \geq \mathbf{A}=\mathbf{A}^{T} \in \mathbb{R}^{N \times N}, u(t), g \in \mathbb{R}^{N}$, B is a diagonal matrix with positive elements and $\delta(t)$ is the Dirac delta function. For example, 1.1 can be obtained from conservative discretization of acoustic wave equation

$$
\nabla \cdot[\sigma \nabla \mathrm{u}(x, t)]-\rho \mathrm{u}_{t t}(x, t)=\mathrm{g}(x) \delta(t),\left.\quad \mathrm{u}\right|_{t<0}=0,
$$

on fine grid, with $x \in \mathbb{R}^{3}$ and variable coefficients $\sigma(x)$ and $\rho(x)$ are respectively medium stiffness and density, in which case $\mathbf{A}$ is the graph-Laplacian operator and elements of $\mathbf{B}$ are masses of the grid cells.

We assume that the reference fine grid operator $\mathbf{A}$ is sufficiently sparse with nontrivial diagonal entries. Its selfadjoint property implies reflective boundary conditions, e.g., Dirichlet or Neumann ones, though our approach allows more general absorbing conditions including the perfectly-matched layer (PML).

We target remote sensing applications, e.g., seismic exploration, where the wavefield is excited by locally supported sources (inputs) $g \in \mathbb{R}^{n}$ and measured by locally supported receiver weight distributions $q \in \mathbb{R}^{n}$ (outputs). For simplicity we assume that they are approximated by discrete $\delta$-functions (columns of the identity matrix).

\footnotetext{
* Schlumberger-Doll Research Center, Cambridge, MA, USA 02139

${ }^{\dagger}$ University of Houston, Houston, TX, USA 77004
} 
In addition, solution of (1.1) is required as a convolution with a waveform of limited frequency bandwidth $\left[0, \omega_{\max }\right]$ assuming $\omega_{\max } \ll \sqrt{\|A\|}$. This is consistent with the condition usually required for a good approximation of the continuous problem [26].

To fix the idea, we assume that the reference fine grid operator $\mathbf{A}$ is obtained via second-order finite-differences or finite element discretization. In general, such methods do not require to have a grid conforming to the medium discontinuities and allow (sometimes with some loss of convergence order) to use different homogenization and error correction techniques to handle curvilinear interfaces, e.g., [31, 34, 25]. However, they require many of degrees of freedom per wavelength for accurate enough approximation. In various important applications, such as seismic exploration, this results in a sparse operator $\mathbf{A}$ of dimension of order $10^{6}-10^{9}$ or even higher.

High-order and spectral methods allow to reduce the size $N$ significantly, however, grid (or elements) adaptation conforming to the interfaces is required to maintain high accuracy. Not only this makes gridding technique complicated, but may also worsen Courant-Friedrich-Levy (CFL) condition for explicit time-stepping stability. We also note that parallelization of low-order methods is significantly more efficient, thanks to the sparsity of the resulting $\mathbf{A}$.

Conventional multi-scale (MS) approaches are targeted to applications where the discretization of spatial operators has to be performed over large-scale domain with multiple small-scale heterogeneities and where accurate approximation of fine-scale effects is not required. Variations of MS methods include MS finite elements, or superelements [15, 16, 22, 3], MS finite volume [23] as well as averaging algorithms 31, 34. MS methods allow to capture small-scale effects of the composite media on medium-scale computational grid (so-called coarse grid). Implementation of MS methods is typically split into an off-line part of computing the approximation space at each coarse grid cell and an on-line part of solving the problem discretized on a coarse grid. The first stage is embarrassingly parallel, i.e. each coarse grid cell can be handled independently. The on-line part requires communications between coarse grid cells, however the cost is rather marginal, thanks to low order of approximation at each coarse cell.

MS methods have been successfully applied for solution of elliptic and parabolic PDEs, particularly with application to reservoir simulations. However, for hyperbolic problems the application of low-order MS methods is limited unless the coarse-scale problem is oversampled in terms of degrees of freedom per wavelength. Likewise, conventional averaging techniques can be applied only when coarse cells are much smaller compared to wavelength. Therefore, high-order MS methods are required for accurate discretization of spatial operators in wave problems. First spectrally accurate multi-scale finite element method for wavefield simulations has been developed in [14]. Spectral convergence is achieved by spanning the approximation space for each coarse cell on a specially chosen subspace of solutions of the Dirichlet problem in that cell. Similar to the traditional MS methods, the approximation problems at each coarse grid cell can be solved independently at the off-line stage. However, the communication and arithmetical costs of the on-line part is affected by appearance of fully dense blocks of the size of those subspaces, responsible for approximation of the wave dynamics within each coarse cell and its interaction with the neighbors.

In this work we address the above problem, by extending so-called optimal (spectrally matched) grids, also known in the literature as finite-difference Gaussian qudrature rules [7, 2. This approach yields discretizations with a sparsity pattern of second order finite-difference schemes but with spectral convergence at the corners of the 
rectangular coarse cells. However, the applications of the optimal grids are limited to the media with uniform coarse cells (below we also call them subdomains). The method presented here (first outlined in [11, 10]) avoids this limitation and allows for arbitrary sharp discontinuities within the subdomains. We will call it the multi-scale S-fraction reduced-order model (MSSFROM), because the sparsification is based on Stieltjes continued fraction (a.k.a. S-fraction) representation of the reduced order models (ROMs) of the Neumann-to-Dirichlet maps (NtD) of the subdomains. The layered structure of the S-fraction introduces several hidden layers in the ROM representation, that results in the block-tridiagonal dynamic system within each coarse cell, thus the communication between coarse cells only involves the state variables from the external layers. This is the crucial new feature of the MSSFROM compared to the original MS formulation of [14, 18. To forestall further discussion, we can refer to Figure 6.2 for graphical introduction of the MSSFROM concept. In fact, this concept can be traced back to an approach to synthesis of complex networks known in electrical engineering and control communities at least from 1960's, when passive electrical networks with desired properties are assembled via a system of passive sub-networks (filters) obtaining via rational approximation of some a priori given impedances, e.g., see [28].

Our starting point is a large stiffness matrix A (of graph-Laplacian type) and a diagonal mass matrix $\mathbf{B}$ obtained from the reference PDE discretization on a fine grid. We begin the first, off-line stage by partitioning the fine computational grid into multiple subdomains and constructing the corresponding partitions of operators $\mathbf{A}$, $\mathbf{B}$ using a divide-and-conquer graph partitioning algorithm. Hereafter we also refer to the subdomains as the coarse grid cells, by analogy with conventional MS methods. For each coarse grid cell we separate the interior and boundary nodes and reduce the entire full-scale problem to the problem on the boundaries of coarse grid cells. The elimination of interior degrees of freedom and further conjugation of adjacent coarse cells is performed in terms of discrete Neumann-to-Dirichlet maps (NtD), defined via Schur complement-like matrices.

The NtD in the frequency or time domain can be viewed as a multi-input/multioutput (MIMO) transfer function of a dynamical system. Model order reduction theory provides well-developed tools to construct low-dimensional approximations of such functions. In this work we adopt the ideas of frequency-limited model reduction that targets an a priori chosen frequency-range [19]. It allows to incorporate the information on limited signal bandwidth into the construction of optimal rational approximant of the transfer function. The obtained reduced-order model (ROM) has significantly fewer degrees of freedom, however, its matrix representation is fully dense. This is similar to replacing a low-order discretization with a spectral one. We show then that the obtained coarse cell ROM can be expressed in block tridiagonal form thanks to layered structure of the S-fraction representation. The advantage of such transformation is twofold. First, the conjugation of the adjacent cells results in the network approximation with matrix coefficients of the size of the S-fraction layers. Second, at on-line stage, exclusion of hidden layers of the S-fraction from the conjugation condition allows to minimize not only the cost of algebraic operations with reduced operator, but also the cost of communication between adjacent cells. The latter is highly important for implementation on high-performance computing (HPC) architectures where the communication cost often dominates the cost of computation.

The paper is organized as follows. In section 2 we partition the fine-grid dynamical system into the subproblems on coarse cells. In section 3 we eliminate the interior 
unknowns at each coarse cell, and the problem is reformulated in terms of Neumannto-Dirichlet maps (boundary transfer functions) of the coarse cells. Then, in section 4 we discuss in details the model reduction technique used for the approximation of Neumann-to-Dirichlet maps of coarse cells. In particular, we compress the inputs and outputs (see section 4.1) and then construct a reduced-order model of the transfer function (see section 4.2). In section 5, we obtain sparse (block-tridiagonal) realization of the the coarse cell ROM by equivalently transforming it to a matrix S-fraction. The global multi-scale ROM is obtained by coupling these sparse ROM realizations via conjugation conditions in section 6. Then we summarize our approach in section 7 and in section 8we prove Stieltjes property (stieltjesness) of the obtained multi-scale ROM, that implies its stability and energy conservation of its minimal realization. A number of numerical examples showing efficiency of our approach are presented in section 9 . In the appendix we describe in more detail the coarse cell partitioning algorithm for graph-Laplacian type of operators (Appendix A) and the efficient computation of the S-fraction coefficients via the block-Lanczos algorithm (Appendix C).

2. Grid partitioning and operator splitting. The very first step of the offline stage is to partition the reference fine grid into the subdomains (coarse cells) and obtain the corresponding splitting of the fine grid stiffness matrix $\mathbf{A}$ and mass matrix B. For this we will need the following elementary definitions from graph theory.

Let $a_{k l}, 1 \leq k, l \leq N$ be the elements of $\mathbf{A}$ and $\Omega$ be the nodal set of the graph associated with $\mathbf{A}$, i.e., the set of reference fine grid nodes. The nodes $k, l \in \Omega$ are called adjacent iff $a_{k l} \neq 0$. For any $\Omega \subset \Omega$ we call its neighborhood $\mathcal{A}(\Omega)$ the set of all adjacent nodes of $\Omega$. If $\mathbf{A}$ is a full matrix, then $\forall \Omega \subset \Omega \mathcal{A}(\Omega)=\Omega$. In the other extreme case of diagonal $\mathbf{A}$, obviously, $\mathcal{A}(\Omega)=\stackrel{\Omega}{\Omega}$.

We assume, that $\Omega$ can be partitioned into $N_{c}\left(N_{c} \geq 2\right)$ nonempty (possibly intersecting) subsets $\Omega_{i}$ with $N_{i}$ nodal points (fine grid nodes) in each that we refer to as the subdomains or coarse cells. For all $i \neq j$ the subdomains $\Omega_{i}$ and $\Omega_{j}$ are coupled via their intersection $\Gamma_{i j}=\Omega_{i} \cap \Omega_{j}$, i.e.,

$$
\Gamma_{i j}=\mathcal{A}\left(\stackrel{\Omega}{i}_{i}\right) \cap \mathcal{A}\left(\stackrel{\Omega}{j}_{j}\right)
$$

where $\stackrel{\circ}{\Omega}_{i}=\Omega_{i} \backslash \Gamma_{i}$ and $\Gamma_{i}=\bigcup_{j \neq i} \Gamma_{i j}$. We will call $\stackrel{\Omega}{\Omega}_{i}$ and $\Gamma_{i}$ respectively the interior and the boundaries of subdomain $\Omega_{i}$.

We will limit our consideration to the case when the outputs and inputs and are supported on the partitioning "skeleton" $\Gamma=\bigcup_{j=1}^{N_{c}} \Gamma_{j}$, i.e.,

$$
\left.q\right|_{\Omega}=0,\left.g\right|_{\Omega}=0, \quad \stackrel{\circ}{\Omega}=\bigcup_{j=1}^{N_{c}} \stackrel{\circ}{\Omega}_{j}
$$

REMARK 1. In addition to its main objective to minimize the arithmetical cost and communications, our domain partitioning algorithm is constrained by condition (2.2) with inputs $g$ and outputs $q$ (approximated by discrete $\delta$-functions) given a priori.

We define prolongation operators $\mathbf{P}_{i} \in \mathbb{R}^{N \times N_{i}}$ via the action on $x \in \mathbb{R}^{N^{i}}$ as

$$
\left(\mathbf{P}_{i} x\right)_{k}= \begin{cases}x_{k}, & \text { if } k \in \Omega_{i} \\ 0, & \text { otherwise }\end{cases}
$$


We split the operators $\mathbf{A}$ and $\mathbf{B}$ respectively on $\mathbf{A}_{i}, \mathbf{B}_{i} \in \mathbb{R}^{N_{i} \times N_{i}}$ defined on subdomains $\Omega_{i}$ so that for any $\omega \in \mathbb{C}$ we have

$$
\mathbf{A}+\omega^{2} \mathbf{B}=\sum_{i=1}^{N_{c}} \mathbf{P}_{i}\left(\mathbf{A}_{i}+\omega^{2} \mathbf{B}_{i}\right) \mathbf{P}_{i}^{T} .
$$

We require $\mathbf{A}_{i}$ and $\mathbf{B}_{i}$ to be nonnegative and positive definite matrices respectively. Let us consider some simple examples, illustrating such splitting.

Example 1. Consider a $1 D$ wave equation

$$
u_{x x}-u_{t t}=g
$$

on an interval $(-1,1)$ with homogeneous Dirichlet boundary conditions on $\partial \Omega$. Let the equation be discretized on a uniform finite-difference grid $\left\{x_{i}=i h\right\}_{i=-n}^{n}$ using a second-order scheme in space $\mathbf{A} u-u_{t t}=g$, where

$$
(\mathbf{A} u)_{k}=\frac{1}{h}\left(\frac{u_{k+1}-u_{k}}{h}-\frac{u_{k}-u_{k-1}}{h}\right), \quad u_{-n}=u_{n}=0 .
$$

Let $\Omega_{1}=\{k \mid-n \leq k \leq 0\}, \Omega_{2}=\{k \mid 0 \leq k \leq n\}$ and, consequently, $\Gamma=\{k=0\}$. The matrix $\mathbf{A} \in \mathbb{R}^{(2 n-1) \times(2 n-1)}$ in this case is diagonally dominant.

The split operators are defined as follows:

$$
\begin{aligned}
& \left(\mathbf{A}_{1} u\right)_{k}=(\mathbf{A} u)_{k}, \quad k<0, \\
& \left(\mathbf{A}_{1} u\right)_{0}=-\frac{1}{h}\left(\frac{u_{0}-u_{-1}}{h}\right), \\
& \left(\mathbf{A}_{2} u\right)_{k}=(\mathbf{A} u)_{k}, \quad k>0, \\
& \left(\mathbf{A}_{2} u\right)_{0}=\frac{1}{h}\left(\frac{u_{1}-u_{0}}{h}\right) .
\end{aligned}
$$

Moreover, since $a_{00}=-\sum_{k \neq 0} a_{0 k}$, this is the unique splitting with nonnegative definite $\mathbf{A}_{i}$ for the given partitioning.

The splitting of $\mathbf{B}$ (which is the identity matrix here) into $\mathbf{B}_{1} \in \mathbb{R}^{n \times n}$ and $\mathbf{B}_{2} \in$ $\mathbb{R}^{n \times n}$ is not unique. Indeed, it can be chosen as

$$
\begin{array}{ll}
\left(\mathbf{B}_{1} u\right)_{k}=1, & k<0, \quad\left(\mathbf{B}_{1} u\right)_{0}=\beta, \\
\left(\mathbf{B}_{2} u\right)_{k}=1, & k>0, \quad\left(\mathbf{B}_{2} u\right)_{0}=1-\beta,
\end{array}
$$

with any $\beta \in(0,1)$. From the discretization point of view, this non-uniqueness is caused by an ambiguity in choosing the dual grids nodes, i.e. the nodes to which the approximation of the derivative is assigned. This is equivalent to discretizing 2.5) in $\Omega_{1}$ and $\Omega_{2}$ independently with Neumann conditions at $\Gamma$, with the first dual steps scaled by $\beta$ and $1-\beta$ respectively. We choose $\beta=\frac{1}{2}$ to approximately balance the condition numbers of the splitted matrix pencils.

EXAMPLE 2. Consider a $2 D$ wave equation

$$
\Delta u-u_{t t}=g
$$

in a rectangular domain $(-1 ; 1) \times(0 ; 1)$ with homogeneous Dirichlet boundary conditions on $\partial \Omega$. Let the equation be discretized on a uniform finite-difference grid

$$
\left\{\left(x_{p}, y_{q}\right) \mid x_{k}=p h, y_{l}=q h\right\}_{k=-n, l=0}^{n, n}
$$


using a second-order scheme in space $\mathbf{A} u-u_{t t}=g$, where the action of the symmetric operator $\mathbf{A} \in \mathbb{R}^{(2 n-1)(n-1) \times(2 n-1)(n-1)}$ is defined as

$$
\begin{aligned}
(\mathbf{A} u)_{p, q} & =\frac{1}{h}\left(\frac{u_{p+1, q}-u_{p, q}}{h}-\frac{u_{p, q}-u_{p-1, q}}{h}\right) \\
& +\frac{1}{h}\left(\frac{u_{p, q+1}-u_{p, q}}{h}-\frac{u_{p, q}-u_{p, q-1}}{h}\right)
\end{aligned}
$$

with boundary conditions $u_{p, 0}=u_{p, n}=u_{-n, q}=u_{n, q}=0$.

We begin with the two-domain partitioning of

$$
\Omega=\{(p, q) \mid p=-n, \ldots, n ; q=0, \ldots, n\}
$$

and define the splitting as follows. Let

$$
\Omega_{1}=\{(p, q) \mid-n \leq p \leq 0\}, \quad \Omega_{2}=\{(p, q) \mid 0 \leq p \leq n\}
$$

and, consequently, $\Gamma=\{(0, q) \mid q=0, \ldots, n\}$. The split operators are defined as

$$
\begin{aligned}
\left(\mathbf{A}_{1} u\right)_{p, q} & =(\mathbf{A} u)_{p, q}, \quad p<0, \\
\left(\mathbf{A}_{1} u\right)_{0, q} & =-\frac{1}{h}\left(\frac{u_{0, q}-u_{-1, q}}{h}\right)+\frac{0.5}{h}\left(\frac{u_{0, q+1}-u_{0, q}}{h}-\frac{u_{0, q}-u_{0, q-1}}{h}\right)
\end{aligned}
$$

with boundary conditions $u_{p, 0}=u_{p, n}=u_{-n, q}=0$ and

$$
\begin{aligned}
\left(\mathbf{A}_{2} u\right)_{p, q} & =(\mathbf{A} u)_{p, q}, \quad k>0, \\
\left(\mathbf{A}_{2} u\right)_{0, q} & =\frac{1}{h}\left(\frac{u_{1, q}-u_{0, q}}{h}\right)+\frac{0.5}{h}\left(\frac{u_{0, q+1}-u_{0, q}}{h}-\frac{u_{0, q}-u_{0, q-1}}{h}\right)
\end{aligned}
$$

with boundary conditions $u_{p, 0}=u_{p, n}=u_{n, q}=0$;

$$
\begin{aligned}
& \left(\mathbf{B}_{1} u\right)_{p, q}=1, \quad p<0, \quad\left(\mathbf{B}_{1} u\right)_{0, q}=\frac{1}{2} \\
& \left(\mathbf{B}_{2} u\right)_{p, q}=1, \quad p>0, \quad\left(\mathbf{B}_{2} u\right)_{0, q}=\frac{1}{2} .
\end{aligned}
$$

In this scenario $\mathbf{A}$ is diagonally dominant, and so is its splitting $\mathbf{A}_{1}, \mathbf{A}_{2}$.

EXAMPLE 3. Now let us consider a multi-domain splitting of $\mathbf{A}, \mathbf{B}$ from Example 2 on a regular square coarse grid. To distinguish the coordinate directions explicitly, we use here the notation with two indices. We define for $i=1, \ldots, N_{c}^{x}$ and $j=1, \ldots, N_{c}^{y}$ the partitioning

$$
\Omega_{i, j}=\left\{(p, q) \mid p=n_{x}(i-1)-n+1, \ldots, n_{x} i-n, q=n_{y}(j-1)+1, \ldots, n_{y} j\right\}
$$

of $\Omega=\{(p, q) \mid p=-n, \ldots, n, q=1, \ldots, n\}$. Here $n_{x} N_{c}^{x}=2 n, n_{y} N_{c}^{y}=n$ and $N_{c}^{x} N_{c}^{y}=N_{c}$ where $N_{c}^{x}, N_{c}^{y}$ are the numbers of coarse cells (subdomains) in each direction and $n_{x}, n_{y}$ are the dimensions of coarse cells. Partitioning skeleton is given by

$$
\begin{array}{r}
\Gamma=\left\{\left(n_{x} i-n, j\right) \mid i=1, \ldots, N_{c}^{x}-1, j=1, \ldots, n\right\} \cup \\
\left\{\left(i, n_{y} j\right) \mid i=-n, \ldots, n, j=1, \ldots, N_{c}^{y}-1\right\}
\end{array}
$$


We split the operators $\mathbf{A}$ and $\mathbf{B}$ as

$$
\begin{aligned}
\left(\mathbf{A}_{i, j} u\right)_{p, q}= & \frac{\gamma_{x}}{h}\left(\alpha_{x}^{+} \frac{u_{p+1, q}-u_{p, q}}{h}-\alpha_{x}^{-} \frac{u_{p, q}-u_{p-1, q}}{h}\right)+ \\
& \frac{\gamma_{y}}{h}\left(\alpha_{y}^{+} \frac{u_{p, q+1}-u_{p, q}}{h}-\alpha_{y}^{-} \frac{u_{p, q}-u_{p, q-1}}{h}\right), \\
\left(\mathbf{B}_{i, j} u\right)_{p, q}= & \beta_{x} \beta_{y} u_{p, q},
\end{aligned}
$$

for $(p, q) \in \Omega_{i, j}$, where the coefficients $\alpha_{x}^{+}, \alpha_{x}^{-}, \alpha_{y}^{+}, \alpha_{y}^{-}, \gamma_{x}, \gamma_{y}, \beta_{x}, \beta_{y}$ are equal to 1 for $(k, l) \notin \Gamma$ and are defined as follows otherwise

- if $p=n_{x}(i-1)-n$ then $\alpha_{x}^{+}=1, \alpha_{x}^{-}=0, \gamma_{y}=0.5, \beta_{x}=0.5$

- if $p=n_{x} i-n$ then $\alpha_{x}^{+}=0, \alpha_{x}^{-}=1, \gamma_{y}=0.5, \beta_{x}=0.5$

- if $n_{x}(i-1)-n<p<n_{x} i-n$ then $\alpha_{x}^{+}=\alpha_{x}^{-}=0.5, \gamma_{y}=1, \beta_{x}=1$

- if $q=n_{y}(j-1)$ then $\alpha_{y}^{+}=1, \alpha_{y}^{-}=0, \gamma_{x}=0.5, \beta_{y}=0.5$

- if $q=n_{y} j$ then $\alpha_{y}^{+}=0, \alpha_{y}^{-}=1, \gamma_{x}=0.5, \beta_{y}=0.5$

- if $n_{y}(j-1)<q<n_{y} j$ then $\alpha_{y}^{+}=\alpha_{y}^{-}=0.5, \gamma_{x}=1, \beta_{y}=1$

To optimize the cost and simplify the partitioning, we usually consider partitioning into regular coarse grids with cubic cells $\Omega_{i}$ (corresponding to actual subdomains of the computational domain), similar to the one shown in Example 3 . Generally, such partitioning and splitting is an intuitively obvious procedure for $\mathbf{A}$ obtained from a discretization of an elliptic operator in regular domains with regular fine grids.

However, for more complicated discretizations and vectorial PDEs (e.g., elasticity), one can perform graph partitioning via Algorithm 4 from Appendix A. By construction it yields diagonal $\mathbf{B}_{i} \succ 0$ and for (non-strictly) diagonally dominant $\mathbf{A}$ it guarantees (non-strict) diagonal dominance of $\mathbf{A}_{i}$ (see Proposition 2 in Appendix A.

3. Elimination of interior nodes via boundary transfer functions. In the previous section we defined the interior $\Omega_{i}$ and boundary nodes $\Gamma_{i}$ for each subdomain $i=1, \ldots, N_{c}$. The next step is to eliminate the interior nodes $\Omega_{i}$. This is done via a boundary transfer function, a Neumann-to-Dirichlet map defined at the boundaries $\Gamma_{i}$. A natural setting to define boundary transfer functions is the frequency (Laplace) domain.

We consider the Laplace-domain counterpart of (3.1) (with some abuse of notation we denote the time and frequency-domain solutions by $u$ )

$$
\mathbf{A} u+\omega^{2} \mathbf{B} u=g
$$

with Laplace frequency $\omega$ satisfying the condition

$$
-\omega^{2} \in \mathbb{C} \backslash\left(\mathbb{R}_{-} \cup\{0\}\right) .
$$

This condition assures non-singularity of $\mathbf{A}+\omega^{2} \mathbf{B}$ and $\mathbf{A}_{i}+\omega^{2} \mathbf{B}_{i}, i=1, \ldots, N_{c}$. From 2.4 we obtain

$$
\sum_{i=1}^{N_{c}} \mathbf{P}_{i}\left(\mathbf{A}_{i}+\omega^{2} \mathbf{B}_{i}\right) \mathbf{P}_{i}^{T} u=g .
$$

We define the prolongation operator $\mathbf{P}_{i}^{\Gamma} \in \mathbb{R}^{N_{i} \times K_{i}}$ from $\Gamma_{i}$ to $\Omega_{i}$ as

$$
\left(\mathbf{P}_{i}^{\Gamma} x\right)_{k}=\left\{\begin{array}{ll}
x_{k}, & \text { if } k \in \Gamma_{i} \\
0, & \text { otherwise }
\end{array} \quad, \quad \text { for } x \in \mathbb{R}^{K_{i}} .\right.
$$


Here we assume that $N_{i} \gg K_{i}$, where $K_{i}$ is the the number of nodes in $\Gamma_{i}$. This assumption is targeted to the case when $\mathbf{A}$ is obtained via a low order discretization of a second order elliptic PDE operator or system. In particular, the case of second order discretizations on regular grids yields $\Gamma_{i}$ that are one reference fine grid node "thick", see Example 3 .

Using 2.2 we eliminate the interior nodes from 3.3 , by transforming it to

$$
\sum_{i=1}^{N_{c}} \mathbf{P}_{i} \mathbf{P}_{i}^{\Gamma}\left(\mathbf{F}_{i}\left(\omega^{2}\right)\right)^{-1}\left(\mathbf{P}_{i} \mathbf{P}_{i}^{\Gamma}\right)^{T} u=g
$$

where

$$
\mathbf{F}_{i}\left(\omega^{2}\right)=\mathbf{P}_{i}^{\Gamma^{T}}\left(\mathbf{A}_{i}+\omega^{2} \mathbf{B}_{i}\right)^{-1} \mathbf{P}_{i}^{\Gamma} \in \mathbb{C}^{K_{i} \times K_{i}}
$$

is the so-called matrix transfer function, a.k.a. Neumann-to-Dirichlet map, Weyl or impedance matrix valued function which is closely related to Schur complements of $\Omega_{i}$. The stieltjesness of $\mathbf{F}_{i}$ (more precisely of its equivalent transform $\mathbf{F}_{i}\left(\omega^{2}\right)$ ) will play a fundamental role in our further derivations. We will use the following definition of the Stieltjes matrix-valued functions introduced by Mark Krein for the scalar case, e.g. see [4, 17, 1].

Definition 1. Let $\mathbf{F}: \mathbb{C} \backslash(0,+\infty) \rightarrow \mathbb{C}^{p \times p}$. Then $\mathbf{F}$ is called a $(0,+\infty)$-Stieltjes function of order $p$ if $\mathbf{F}$ satisfies the following three conditions:

1. $\mathbf{F}$ is holomorphic in $\mathbb{C} \backslash(0,+\infty)$.

2. For all $z$ with $\Im z>0$ the matrix $\Im(\mathbf{F})$ is non-negative definite Hermitian.

3. For all $z \in(-\infty, 0)$ the matrix $\mathbf{F}(z)$ is non-negative definite Hermitian.

The transfer function can be written via the spectral decomposition of the matrix pencil $\left(\mathbf{A}_{i}, \mathbf{B}_{i}\right)$ as

$$
\mathbf{F}_{i}\left(\omega^{2}\right)=\sum_{l=1}^{N_{i}} \frac{V_{l}^{i} V_{l}^{i^{T}}}{\lambda_{l}^{i}+\omega^{2}}
$$

were $\lambda_{l}^{i} \in \mathbb{R}_{-}$are the eigenvalues of matrix pencil $\left(\mathbf{A}_{i}, \mathbf{B}_{i}\right)$ and $V_{l}^{i} \in \mathbb{R}^{K_{i}}$ are the restrictions of the corresponding eigenvectors on $\Gamma_{i}$. It is easy to see that $\mathbf{F}_{i}\left(\omega^{2}\right)$ satisfies all three conditions of Definition 1, so it is the Stieltjes matrix valued function of $\omega^{2}$. Moreover, it can be easily shown via the Schur complement formula, that $\left(\mathbf{F}_{i}\left(\omega^{2}\right)\right)^{-1}$ is linear with respect to $\left(\AA_{i}+\omega^{2} \stackrel{\circ}{\mathbf{B}}_{i}\right)^{-1}$, where $\AA_{i} \leq 0$ and $\stackrel{\circ}{\mathbf{B}}_{i}>0$ are the diagonal blocks respectively of $\mathbf{A}_{i}$ and $\mathbf{B}_{i}$ (equivalently, of $\mathbf{A}$ and $\mathbf{B}$ ) corresponding to $\Omega_{i}$. This allows to replace non-negative definiteness with positive-definiteness in conditions 2 and 3 of Definition 1 , that yields the equivalence of 3.3 and 3.5 .

Denote

$$
\left.u\right|_{\Gamma_{i}}=\mathbf{P}_{i}^{\Gamma^{T}} \mathbf{P}_{i}^{T} u \in \mathbb{R}^{K_{i}}
$$

where $\mathbf{P}_{i}^{\Gamma^{T}} \mathbf{P}_{i}{ }^{T}$ is obviously the restriction operator from $\Omega$ to $\Gamma_{i}$. Then we can equivalently rewrite (3.5) in terms of the boundary restrictions of $u$ as

$$
\left.\sum_{i=1}^{N_{c}} \mathbf{P}_{i} \mathbf{P}_{i}^{\Gamma}\left(\mathbf{F}_{i}\left(\omega^{2}\right)\right)^{-1} u\right|_{\Gamma_{i}}=g
$$

For $N_{c}>2$ there may exist a nonempty "corner" set $\Gamma^{\cap}=\bigcup_{i} \bigcup_{j \neq j^{\prime}} \Gamma_{i j} \cap \Gamma_{i j^{\prime}}$. 
In the case of regular partitioning, similar to the one described in the Example 3 , dimension of such set is $o\left(\sum_{i=1}^{N c} K_{i}\right)$. We also note that from a topological point of view the "corner" set in $3 D$ regular cells includes not only corners but also the entire edges. To simplify the derivation, we consider the case with empty "corner" set

$$
\Gamma^{\cap}=\emptyset \text {. }
$$

The fine grid discretization can be modified to satisfy such condition by introducing hanging nodes and removing nodes from $\Gamma^{n}$ (see Appendix B). We show how it works explicitly for example 3 .

EXAMPLE 4. Let us consider the removal of the corner set

$$
\Gamma^{\cap}=\left\{\left(n_{x} i-n, n_{y} j\right) \mid i=1, \ldots, N_{c}^{x}-1, j=1, \ldots, N_{c}^{y}-1\right\}
$$

of the operator from Example 3 . For each $(p ; q) \in \Gamma^{\cap}$, we introduce four hanging nodes $\left(p, q^{u}\right),\left(p, q^{l}\right),\left(p^{r}, q\right),\left(p^{l}, q\right)$ and replace $(\mathbf{A} u)_{p, q}$ and $(\mathbf{B} u)_{p, q}$ by

$$
\begin{aligned}
& \left(\mathbf{A}^{\prime} u\right)_{p, q^{u}}=\frac{1}{2 h}\left(\frac{u_{p+1, q^{u}}-u_{p, q^{u}}}{h}-\frac{u_{p, q^{u}}-u_{p-1, q^{u}}}{h}\right)+\frac{1}{h}\left(\frac{u_{p, q^{u}+1}-u_{p, q^{u}}}{h}\right), \\
& \left(\mathbf{B}^{\prime} u\right)_{p, q^{u}}=0.5 u_{p, q^{u}}, \\
& \left(\mathbf{A}^{\prime} u\right)_{p, q^{l}}=\frac{1}{2 h}\left(\frac{u_{p+1, q^{l}}-u_{p, q^{l}}}{h}-\frac{u_{p, q^{l}}-u_{p-1, q^{l}}}{h}\right)+\frac{1}{h}\left(\frac{u_{p, q^{l}-1}-u_{p, q^{l}}}{h}\right), \\
& \left(\mathbf{B}^{\prime} u\right)_{p, q^{l}}=0.5 u_{p, q^{l}}, \\
& \left(\mathbf{A}^{\prime} u\right)_{p^{r}, q}=\frac{1}{h}\left(\frac{u_{p^{r}+1, q}-u_{p^{r}, q}}{h}\right)+\frac{1}{2 h}\left(\frac{u_{p^{r}, q+1}-u_{p^{r}, q}}{h}-\frac{u_{p^{r}, q}-u_{p^{r}, q-1}}{h}\right) \\
& \left(\mathbf{B}^{\prime} u\right)_{p^{r}, q}=0.5 u_{p^{r}, q}, \\
& \left(\mathbf{A}^{\prime} u\right)_{p^{l}, q}=\frac{1}{h}\left(\frac{u_{p^{l}-1, q}-u_{p^{l}, q}}{h}\right)+\frac{1}{2 h}\left(\frac{u_{p^{l}, q+1}-u_{p^{l}, q}}{h}-\frac{u_{p^{l}, q}-u_{p^{l}, q-1}}{h}\right) \\
& \left(\mathbf{B}^{\prime} u\right)_{p^{l}, q}=0.5 u_{p^{l}, q} .
\end{aligned}
$$

$\mathbf{B}^{\prime}$ is diagonal and $\mathbf{A}^{\prime}$ is assumed to be symmetric: we define its action on vectors with non-zero elements at hanging node $(p ; q)$ by symmetry via $\left(\mathbf{A}^{\prime} u\right)_{p, q}$. In Figure 3.1 we show the modified graph of $\mathbf{A}$ corresponding to the partitioning from Example 3 with the corner set removed.

The same approach can be straightforwardly extended to the case of $3 D$ (and multidimensional) Cartesian grids, where "corner" set also include the edges of the coarse cells. For regular enough solutions with uniformly bounded first differences such a modification, obviously leads to $O\left(\frac{b_{k k}}{\operatorname{Tr} B}\right)$ error, where $\frac{b_{k k}}{\operatorname{Tr} B}$ is the relative mass of the corner point. When both the fine and coarse grids are uniform Cartesian, the total relative mass of the corner points is inversely proportional to the coarse cell diameter squared. Thus, for large enough coarse cells removal of the corner points adds the error, consistent with the second order discretization error on the fine grid. In all our numerical experiments this modification results in a negligible error, however solution correction can be made at each time step, if needed (see Remark 6).

Introducing a small error and considering the case $(3.8)$ we can simplify the system (3.7) to

$$
\left.\mathbf{P}_{i j}^{T}\left(\mathbf{F}_{i}\left(\omega^{2}\right)\right)^{-1} u\right|_{\Gamma_{i}}+\left.\mathbf{P}_{j i}^{T}\left(\mathbf{F}_{j}\left(\omega^{2}\right)\right)^{-1} u\right|_{\Gamma_{j}}=\left.g\right|_{\Gamma_{i j}}, \quad \forall(i, j): i \neq j, \Gamma_{i j} \neq \emptyset,
$$

where $\mathbf{P}_{i j}^{T} \in \mathbb{R}^{K_{i j} \times K_{i}}$ is the restriction operator from $\Gamma_{i}$ to $\Gamma_{i j}$ and $K_{i j}$ is the dimension of $\Gamma_{i j}$. 

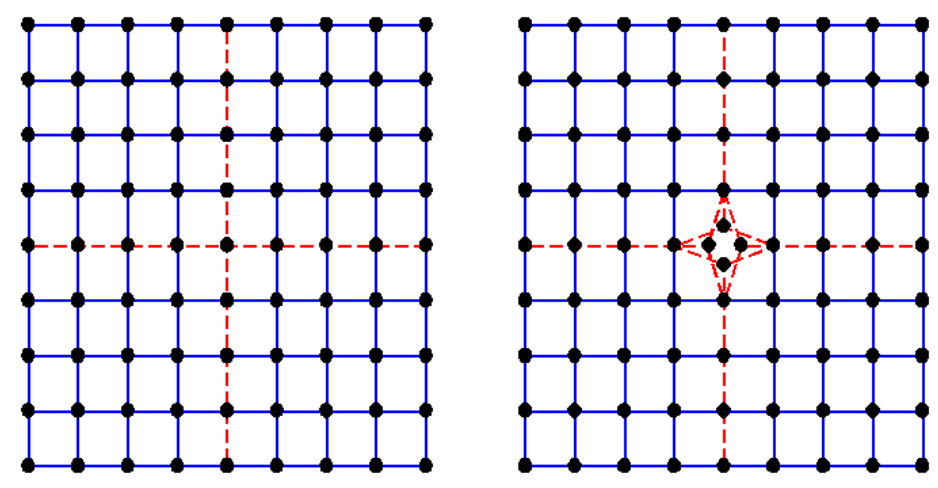

FIG. 3.1. Graph of A from Example 3 (left) and its modification without corner set (right). $\Gamma$ is shown by red lines. $\Gamma^{\cap}$ consists of one node (center node on the left plot).

4. Reduced order models on subdomains. After the partitioning of $\Omega$ and the corresponding splitting of $\mathbf{A}, \mathbf{B}$ is computed and the matrix transfer functions (3.6) are defined on the subdomain boundaries $\Gamma_{i}$, the next point to address is the efficient computation of transfer functions $\mathbf{F}_{i}\left(\omega^{2}\right), i=1, \ldots, N_{c}$. To this effect we replace the true $\mathbf{F}_{i}\left(\omega^{2}\right)$ with reduced-order models. The complexity of the matrixvalued transfer function can be described by its McMillan degree, i.e., the dimension of the equivalent minimal realization via the first order dynamical system 29. Thus, the McMillan degree of the exact transfer function $\mathbf{F}_{i}\left(\omega^{2}\right)$ is generally equal to $2 N_{i}$.

Model order reduction is a two step process.

First, in section 4.1 we consider the reduction of dimensionality of boundary degrees of freedom from $K_{i}$ to $\widetilde{K}_{i} \ll K_{i}$. This is achieved by projecting $\mathbf{F}_{i}\left(\omega^{2}\right)$ on a tall skinny orthogonal matrix $\mathbf{S}_{i} \in \mathbb{R}^{K_{i} \times \widetilde{K}_{i}}, \mathbf{S}_{i}^{T} \mathbf{S}_{i}=\mathbf{I}$, which is obtained via an approximate proper orthogonal decomposition of the boundary restrictions of solutions $\left.u\right|_{\Gamma_{i}}$. The resulting projected transfer function has the form

$$
\widetilde{\mathbf{F}}_{i}\left(\omega^{2}\right)=\mathbf{S}_{i}^{T} \mathbf{F}_{i}\left(\omega^{2}\right) \mathbf{S}_{i} \in \mathbb{R}^{\widetilde{K}_{i} \times \widetilde{K}_{i}}
$$

Second, in section 4.2 we compute matrix Padé approximant $\widetilde{\mathbf{F}}_{i}^{m}\left(\omega^{2}\right)$ of $\widetilde{\mathbf{F}}_{i}\left(\omega^{2}\right)$ via Padé-Krylov connection satisfying $2 m$ matching conditions.

As we shall see in section 6 , the first step decreases both the communication and arithmetical cost, while the second step mainly reduces the arithmetical cost. As a result of these two steps, we reduce the McMillan degree of the final transfer function from $2 N_{i}$ to $2 m \widetilde{K}_{i}$.

4.1. Proper orthogonal decomposition of boundary solution restrictions. The column space of $\mathbf{S}_{i}$ should produce a good approximation basis for the boundary restrictions of all possible propagative solutions. Optimal compression, in principle, can be obtained via the proper orthogonal decomposition (POD) on $\Gamma=\bigcup_{i=1}^{N_{c}} \Gamma_{i}$ of frequency bounded solutions, or equivalently, via frequency-limited balanced truncation 20]. Here we implemented an approximate approach of $[8$ that is outlined below. 
Following 2], we will concentrate on the propagative part of the solution for the cutoff frequency $\omega_{\max }$, that for $t \geq 0$ is given by spectral decomposition

$$
q^{T} u=\sum_{\lambda_{l} \leq \omega_{\max }^{2}} \frac{\sin \sqrt{\lambda_{l}} t}{\sqrt{\lambda_{l}}} q^{T} z_{l} z_{l}^{T} g,
$$

where $\lambda_{l} \in \mathbb{R}$ and $z_{l} \in \mathbb{R}^{N}, l=1,2, \ldots, N$ are respectively the eigenvalues and the eigenvectors of matrix pencil $(\mathbf{A}, \mathbf{B})$. Accurate approximation of the propagative part also gives reasonably good approximation of the entire solution. e.g., see [2].

The subspace of propagative solution restricted on $\Gamma_{i j}$ can be approximately computed via the frequency-limited Gramian:

$$
G_{i j}^{\omega_{\max }}=\sum_{\lambda_{l} \leq \omega_{\max }^{2}} v_{l} v_{l}^{T} \in \mathbb{R}^{K_{i j} \times K_{i j}}, \quad v_{l}=\left.z_{l}\right|_{\Gamma_{i j}},
$$

here $z_{l}$ are the eigenvectors of matrix pencil $(\mathbf{A}, \mathbf{B})$. We denote by $\mathbf{S}_{i j} \in \mathbb{R}^{M_{i j} \times K_{i j}}$ the matrix of eigenvectors of $G_{i j}^{\omega_{\max }}$ corresponding to the eigenvalues above some truncation threshold $\epsilon>0$. Let the set $J(i)$ be defined as

$$
J(i)=\left\{j \mid j \neq i \text { and } \Gamma_{i j} \neq \emptyset\right\} .
$$

We construct $\mathbf{S}_{i} \in \mathbb{R}^{K_{i} \times \widetilde{K}_{i}}, \widetilde{K}_{i}=\sum_{j \in J(i)} M_{i j}$ as a block diagonal matrix with $\mathbf{S}_{i j}$, $j \in J(i)$ as the diagonal (non-square) blocks, i.e.,

$$
\mathbf{S}_{i} \equiv\left[\begin{array}{cccc}
\mathbf{S}_{i j_{1}} & 0 & 0 & \ldots \\
0 & \mathbf{S}_{i j_{2}} & 0 & \ldots \\
\cdots & 0 & \mathbf{S}_{i j_{3}} & 0 \\
& \cdots & 0 & \mathbf{S}_{i j_{\text {jmax }}}
\end{array}\right],
$$

where $J(i)=\left\{j_{1}, \ldots, j_{\text {jmax }}\right\}$.

Assuming fast enough decay of the eigenvalues of $G_{i j}^{\omega_{\max }}$, it can be shown that

$$
\left\|\mathbf{S}_{i} \widetilde{\mathbf{F}}_{i}\left(\omega^{2}\right) \mathbf{S}_{i}^{T}-\mathbf{F}_{i}\left(\omega^{2}\right)\right\|_{\infty}<O(\epsilon) .
$$

It is known that for large enough $N_{i}$ the Gramian truncation procedure exhibits spectral convergence.

Direct computation via spectral decomposition (4.3) is prohibitively expensive for sufficiently large problems. However, $G_{i j}^{\omega_{\max }}$ can be equivalently rewritten as composition of spectral projection and prolongation operators

$$
\left(\mathbf{P}_{i} \mathbf{P}_{i}^{\Gamma} \mathbf{P}_{i j}\right)^{T} \eta\left(\omega_{\max }^{2} \mathbf{I}-\mathbf{B}^{-1} \mathbf{A}\right) \mathbf{P}_{i} \mathbf{P}_{i}^{\Gamma} \mathbf{P}_{i j},
$$

where $\eta(\lambda)$ is the Heaviside step-function, i.e., $\eta(\lambda)=1$ if $\lambda \geq 0$ and 0 otherwise. We compute spectral projector $\eta\left(\omega_{\max }^{2} \mathbf{I}-\mathbf{B}^{-1} \mathbf{A}\right)$ via optimal (Zolotarev) rational approximations of the sign function, e.g., see [21, 6, 32. The Zolotarev approximant usually requires up to 20 linear shifted solves, that can still be expensive with large $N$. To speed up the Zolotarev algorithm we use an approximate Gramian $\widetilde{G}_{i j}^{\omega_{\max }^{\prime}}$ obtained for the matrix $\widetilde{\mathbf{B}}_{i j}^{-1} \widetilde{\mathbf{A}}_{i j}$ of a smaller dimension but with the same block corresponding to $\Gamma_{i j}$. The low dimensional operator $\widetilde{\mathbf{B}}_{i j}^{-1} \widetilde{\mathbf{A}}_{i j}$ is chosen to get accurate approximation of the Schur complement of full problem at $\Gamma_{i j}$. Complete description of this approach will appear in [8]. 
4.2. Reduced order model for the projected transfer function. Having the compressed set of inputs and outputs $\mathbf{S}_{i}$ in hand, the efficient approximation of the projected transfer function

$$
\widetilde{\mathbf{F}}_{i}\left(\omega^{2}\right)=\mathbf{S}_{i}^{T} \mathbf{P}_{i}^{\Gamma^{T}}\left(\mathbf{A}_{i}+\omega^{2} \mathbf{B}_{i}\right)^{-1} \mathbf{P}_{i}^{\Gamma} \mathbf{S}_{i}
$$

can be obtained using traditional projection-based model reduction methods. These methods seek the ROM of $\widetilde{\mathbf{F}}_{i}\left(\omega^{2}\right)$ by replacing $\mathbf{A}_{i}$ and $\mathbf{B}_{i}$ with projections on some subspace $\mathcal{V}_{m}$. Note that projection subspace $\mathcal{V}_{m}$ may be different for different coarse cells. However, for brevity, we omit the index $i$ of $\mathcal{V}_{m}$. The rational Krylov subspace (RKS) is one of the most popular choices of projection subspaces in model order reduction community, allowing to obtain ROMs as multipoint Padé approximants with exponential convergence rates 33 .

In our numerical examples we stick to block rational Krylov subspace*

$$
\mathcal{V}_{m}=\operatorname{colspan}\left\{\mathbf{P}_{i}^{\Gamma} \mathbf{S}_{i},\left(\mathbf{A}_{i}+s \mathbf{B}_{i}\right)^{-1} \mathbf{P}_{i}^{\Gamma} \mathbf{S}_{i}, \ldots,\left(\mathbf{A}_{i}+s \mathbf{B}_{i}\right)^{-m+1} \mathbf{P}_{i}^{\Gamma} \mathbf{S}_{i}\right\}
$$

with some small (in absolute value) negative shift $s$. This RKS can be implemented by applying a block Lanczos iteration to the pair $\left(\left(\mathbf{A}_{i}+s \mathbf{B}_{i}\right)^{-1}, \mathbf{P}_{i}^{\Gamma} \mathbf{S}_{i}\right)$.

Assumption 1. Here we assume that all $\left(\mathbf{A}_{i}+s \mathbf{B}_{i}\right)^{-k} \mathbf{P}_{i}^{\Gamma} \mathbf{S}_{i}$ are linearly independent for $k=0, \ldots, m-1$, i.e. $\operatorname{dim}\left(\mathcal{V}_{m}\right)=m \widetilde{K}_{i}$. This assumption generally holds for problems arising from discretization of uniformly bounded elliptic PDEs provided $m \widetilde{K}_{i} \ll N_{i}$, but theoretically may break in the case of elliptic systems with degeneration, e.g., in the mix of solid and liquid for the elasticity system. This would require block Lanczos method with deflation, but we did not observe such phenomena in practice.

Note that the computation of $\mathcal{V}_{m}$ requires multiple linear solves with shifted matrix $\mathbf{A}_{i}$ that can be quite costly. However, the cost is alleviated by several factors. First, the computation is only done on small subdomains. Second, the computations for different subdomains are independent of each other, thus they can be performed in parallel without any data exchange. Third, the computation only must be done just once before the time stepping for all sources, what we refer to as the off-line computation. Also, a precomputed Cholesky factorization can be reused for the repeated linear solves.

REMARK 2. Projection on subspace (4.5) is easy to implement and it provides $\widetilde{\mathbf{F}}_{i}^{m}$ as Padé approximants of $\widetilde{\mathbf{F}}_{i}$ as function of $\omega^{2}$ at frequency $s$ with exponential convergence for $\omega^{2} \in \mathbb{C} \backslash \lambda\left[\mathbf{B}_{i}^{-1} \mathbf{A}_{i}\right]$. However, it may not be optimal in terms of the number of degrees of freedom per wavelength. Other model reduction techniques such as time- and/or frequency-limited balanced truncation [20] may be more appropriate for these purposes, however their implementation can be more expensive.

Let $\mathbf{V}_{m} \in \mathbb{R}^{N_{i} \times m \widetilde{K}_{i}}$ be an orthonormal basis for $\mathcal{V}_{m}$. Reduced-order model of the projected transfer function can be obtained as

$$
\widetilde{\mathbf{F}}_{i}^{m}\left(\omega^{2}\right)=\left(\mathbf{S}_{i}^{m}\right)^{T}\left(\mathbf{A}_{i}^{m}+\omega^{2} \mathbf{B}_{i}^{m}\right)^{-1} \mathbf{S}_{i}^{m} \in \mathbb{R}^{\widetilde{K}_{i} \times \widetilde{K}_{i}}
$$

where $\mathbf{A}_{i}^{m}=\mathbf{V}_{m}^{T} \mathbf{A}_{i} \mathbf{V}_{m} \in \mathbb{R}^{m \widetilde{K}_{i} \times m \widetilde{K}_{i}}$ and $\mathbf{B}_{i}^{m}=\mathbf{V}_{m}^{T} \mathbf{B}_{i} \mathbf{V}_{m} \in \mathbb{R}^{m \widetilde{K}_{i} \times m \widetilde{K}_{i}}$ is the projected matrix pencil and $\mathbf{S}_{i}^{m}=\mathbf{V}_{m}^{T} \mathbf{P}_{i}^{\Gamma} \mathbf{S}_{i} \in \mathbb{R}^{m \widetilde{K}_{i} \times \widetilde{K}_{i}}$ is the projected inputoutput.

LEMMA 4.1. Matrix valued function $\widetilde{\mathbf{F}}_{i}^{m}(s)$ is the $(0, \infty)$-Stieltjes function of order $\widetilde{K}_{i}$

${ }^{*}$ See remark 4 for discussion whether to include zero power term $\mathbf{S}_{i}$ in the subspace. 
Proof. Projected matrices $\mathbf{A}_{i}^{m}$ and $\mathbf{B}_{i}^{m}$ are symmetric and respectively nonpositive and positive definite, so their pencil has eigenpairs $\theta_{l}^{i} \in \mathbb{R}_{-}, y_{l}^{i} \in \mathbb{R}^{m \widetilde{K}_{i}}$, $l=1, \ldots, m \widetilde{K}_{i}$. From the spectral decomposition we obtain a partial fraction representation

$$
\widetilde{\mathbf{F}}_{i}^{m}\left(\omega^{2}\right)=\sum_{l=1}^{m \widetilde{K}_{i}} \frac{v_{l} v_{l}^{T}}{\theta_{l}^{i}+\omega^{2}}, \quad v_{l}=P_{i}^{\Gamma^{T}} y_{l}^{i} \in \mathbb{R}^{\widetilde{K}_{i}} .
$$

Obviously, representation 4.7) satisfies all 3 conditions of Definition 1.

Assumption 2. It is known that the McMillan degree of the reduced order model $\widetilde{\mathbf{F}}_{i}^{m}(s)$ (that can also be equivalently defined as twice the minimal number of terms in representation (4.7), [5]) is bounded by twice the dimension of the projection space (in the second order formulation, i.e. by $\left.2 m \widetilde{K}_{i}\right)$. We assume that the actual McMillan degree of $\widetilde{\mathbf{F}}_{i}^{m}(s)$ is equal to this bound, which is what we observed in our experiments given in section 9 for sufficiently large $N_{i}$.

5. Equivalent sparse representations of ROMs. The ROM 4.6 in general involves the projected matrix pencil $\mathbf{A}_{i}^{m}, \mathbf{B}_{i}^{m} \in \mathbb{R}^{m \widetilde{K}_{i} \times m \widetilde{K}_{i}}$ with dense matrices. This leads to high computational costs at the on-line stage when the time stepping is performed with such operators. To reduce the computational cost of the on-line stage we need to compute the equivalent minimal realization of the ROM via a linear timeinvariant dynamic system. In this section we construct a sparse realization of such system, which mimics the structure of a three-point second order finite-difference scheme.

The approach of this section is a direct generalization of the so-called finitedifference quadrature rules a.k.a. spectrally matched or optimal grids [7, 2, from scalar to matrix transfer functions. For brevity, in this section we omit index $i$ assuming that we currently consider a single coarse cell $\Omega_{i}$ only.

The core of our approach is an equivalent representation of $\widetilde{\mathbf{F}}^{m}\left(\omega^{2}\right)$ via matrix Stieltjes continuous fraction (S-fraction) form [4] as

$$
\widetilde{\mathbf{F}}^{m}\left(\omega^{2}\right)=\frac{1}{-\frac{\omega^{2}}{\widehat{\mathbf{L}}^{1}}+\frac{1}{\frac{1}{\mathbf{L}^{1}}+\frac{1}{\ddots+\frac{1}{-\frac{\omega^{2}}{\hat{\mathbf{L}}^{m}}+\mathbf{L}^{m}}}}},
$$

where $\widehat{\mathbf{L}}^{k}, \mathbf{L}^{k} \in \mathbb{R}^{\widetilde{K} \times \widetilde{K}}$ are symmetric positive-definite matrices and the division is understood as matrix inversion. As follows from results of [13, Assumption 2 guarantees the existence and uniqueness of the S-fraction representation (5.1) of Stieltjes $\widetilde{\mathbf{F}}^{m}\left(\omega^{2}\right)$ obtained (as in the previous section) via the matrix Padé approximant.

A direct recursive algorithm transforming $\widetilde{\mathbf{F}}^{m}$ from the partial fraction form 4.7) to the S-fraction form (5.1) is Algorithm 7.2 in [9]. It also can be viewed as a constructive existence proof, that if such a representation exists, it is unique and automatically produces positive definite $\widehat{\mathbf{L}}^{k}, \mathbf{L}^{k}, k=1,2, \ldots, m$ for Stieltjes $\widetilde{\mathbf{F}}^{m}$. Another, more complicated but also more computationally efficient algorithm via the block-Lanczos method is given in Appendix C.

The novelty of our approach is in following original Krein's representation of the scalar S-fraction via a string of point masses and weightless springs, a so-called 
Stieltjes string [24], to represent $\widetilde{\mathbf{F}}^{m}\left(\omega^{2}\right)$ equivalently via a transfer function of a "three-point finite-difference scheme" with matrix coefficients

$$
\begin{aligned}
\mathbf{L}^{1}\left(\mathbf{U}^{2}-\mathbf{U}^{1}\right)+\omega^{2}\left(\widehat{\mathbf{L}}^{1}\right)^{-1} \mathbf{U}^{1} & =\mathbf{I}, \\
\mathbf{L}^{k}\left(\mathbf{U}^{k+1}-\mathbf{U}^{k}\right)-\mathbf{L}^{k-1}\left(\mathbf{U}^{k}-\mathbf{U}^{k-1}\right)+\omega^{2}\left(\widehat{\mathbf{L}}^{k}\right)^{-1} \mathbf{U}^{k} & =\mathbf{0},
\end{aligned}
$$

by introducing fictitious (full rank) matrix variables $\mathbf{U}^{k} \in \mathbb{C}^{\widetilde{K} \times \widetilde{K}}, k=1,2, \ldots, m+1$, with $\mathbf{U}^{m+1}=0$, so that

$$
\widetilde{\mathbf{F}}^{m}\left(\omega^{2}\right)=\mathbf{U}^{1} .
$$

REMARK 3. In the case $\widetilde{K}=1,(5.1$ becomes a scalar $S$-fraction and (5.2)-(5.3) becomes the regular second-order finite-difference scheme on the optimal staggered grid with primary and dual steps $1 / \mathbf{L}^{j}$ and $1 / \widehat{\mathbf{L}}^{j}$ respectively.

For multidimensional problems $(\widetilde{K}>1)$, the columns of $\mathbf{U}^{1}$ give the projections of the solutions on the subdomain boundary, and the columns of $\mathbf{U}^{k}$ for $k>1$ have the same dimension $\widetilde{K}$, i.e., they can be viewed as projections of solutions on some virtual concentric layers inside $\Omega_{i}$ and (5.2 5.3) can be interpreted as a three-point second order discretization in the subdomain interior in the direction normal to these layers. A schematic representation of the of the stencil inside $\Omega_{i}$ is shown in Figure 5.1. The $S$-fraction representation is a known tool in the homogenization theory (e.g., see [27]). Indeed, if subdomain has dimensions much less compared to the wavelength then conventional non-dispersive isotropic or anisotropic effective medium can be constructed. Otherwise, the effective medium has to be frequency-dependent (dispersive). In this contest (5.1) can be viewed as an effective dynamic impedance of the subdomain converted to an equivalent network approximation via (5.2)-(5.4).

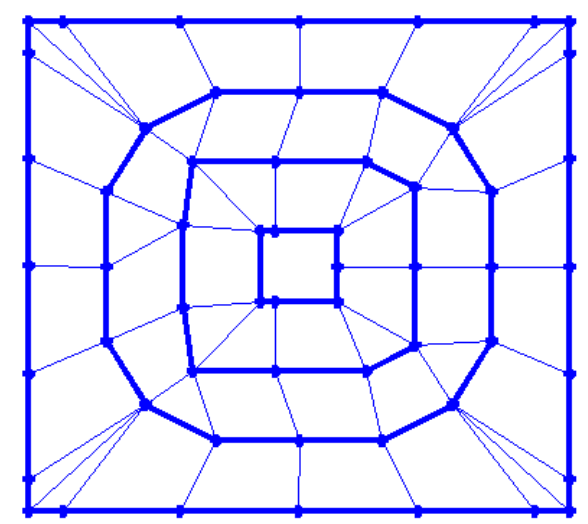

FIG. 5.1. Schematic representation of the stencil for the s-fraction ROM inside single subdomain. Each layer of unknowns is connected with its neighbors only. Here we only outline just some possible nodes and connections: Connection between neighbors is dense in general, i.e., every unknown is one layer is connected with all unknowns inside that layer as well as all unknowns in the neighboring layers; The number of the unknowns at every layer is the same as at the coarse cell boundary, i.e., $\widetilde{K}$, see remark 3 .

6. Boundary conjugation. Construction of the ROM $\widetilde{\mathbf{F}}_{i}^{m}$ and its S-fraction representation (5.1) concludes the off-line stage. At the on-line stage the solutions at 
neighboring subdomains need to be conjugated via the reduced order transfer functions on the shared boundaries. This leads to data exchange between the subdomains. In this section we derive the formulas for solution conjugation which dictate the data to be exchanged.

Replacing the true full-scale transfer function $\mathbf{F}_{i}\left(\omega^{2}\right)$ in $\sqrt{3.9}$ with ROM $\sqrt{5.4}$ we obtain

$$
\widetilde{\mathbf{P}}_{i j}^{T}\left(\widetilde{\mathbf{F}}_{i}^{m}\left(\omega^{2}\right)\right)^{-1} U_{i}^{1}+\widetilde{\mathbf{P}}_{j i}^{T}\left(\widetilde{\mathbf{F}}_{j}^{m}\left(\omega^{2}\right)\right)^{-1} U_{j}^{1}=\widetilde{g}_{i j}
$$

where

$$
\widetilde{g}_{i j}=\left.\mathbf{S}_{i j}^{T} g\right|_{\Gamma_{i j}},
$$

and $\widetilde{\mathbf{P}}_{i j}^{T} \in \mathbb{R}^{\widetilde{K}_{i j} \times \widetilde{K}_{i}}$ is the restriction matrix from the set of reduced inputs-outputs $\mathbf{S}_{i}$ to the set that corresponds to $\mathbf{S}_{i j}$.

Vector variable $U_{i}^{1} \in \mathbb{R}^{\widetilde{K}_{i}}$ (a function of $\omega$ ) has the meaning of the projected approximate solution of 3.9 , i.e.

$$
\left.u\right|_{\Gamma_{i}} \approx \mathbf{S}_{i} U_{i}^{1}
$$

Hence, the solution at receiver $q$ can be approximated by

$$
q^{T} u \approx \sum_{i, j} \widetilde{q}_{i j} \widetilde{u}_{i j}
$$

where

$$
\widetilde{u}_{i j}=\widetilde{\mathbf{P}}_{i j}^{T} U_{i}^{1}
$$

and

$$
\widetilde{q}_{i j}=\left.\mathbf{S}_{i j}^{T} q\right|_{\Gamma_{i j}}
$$

We impose conjugation conditions

$$
\widetilde{u}_{j i}=\widetilde{u}_{i j}
$$

for every two adjacent subdomains $\Omega_{i}$ and $\Omega_{j}$. By construction $\mathbf{S}_{i j}=\mathbf{S}_{j i}$, so 6.3 leads to conjugation for the approximate solutions at $\Gamma_{i j}$. From (5.2) and (5.4) for subdomain $\Omega_{i}$ we obtain

$$
\left(\mathbf{L}_{i}^{1}\left(\mathbf{U}_{i}^{2}-\mathbf{U}_{i}^{1}\right)\right)+\omega^{2}\left(\widehat{\mathbf{L}}_{i}^{1}\right)^{-1} \mathbf{U}_{i}^{1}=\left(\widetilde{\mathbf{F}}_{i}^{m}\left(\omega^{2}\right)\right)^{-1} \mathbf{U}_{i}^{1} .
$$

The same holds for $\Omega_{j}$. As it was mentioned above, matrices $\mathbf{U}_{i}^{k}$ are of full rank, so for $\Omega_{i}$ we can write

$$
\left(\mathbf{L}_{i}^{1}\left(U_{i}^{2}-U_{i}^{1}\right)\right)+\omega^{2}\left(\widehat{\mathbf{L}}_{i}^{1}\right)^{-1} U_{i}^{1}=\left(\widetilde{\mathbf{F}}_{i}^{m}\left(\omega^{2}\right)\right)^{-1} U_{i}^{1}
$$

where $U_{i}^{2} \in \mathbb{R}^{\widetilde{K}_{i}}$. The same relation holds for the $\Omega_{j}$. Then equation 6.1 can be rewritten as

$$
\begin{aligned}
\widetilde{\mathbf{P}}_{i j}^{T}\left(\mathbf{L}_{i}^{1}\left(U_{i}^{2}-U_{i}^{1}\right)\right)+\widetilde{\mathbf{P}}_{j i}^{T}\left(\mathbf{L}_{j}^{1}\left(U_{j}^{2}-U_{j}^{1}\right)\right)+ \\
\omega^{2}\left(\widetilde{\mathbf{P}}_{i j}^{T}\left(\widehat{\mathbf{L}}_{i}^{1}\right)^{-1} U_{i}^{1}+\widetilde{\mathbf{P}}_{j i}^{T}\left(\widehat{\mathbf{L}}_{j}^{1}\right)^{-1} U_{j}^{1}\right)=\widetilde{g}_{i j} .
\end{aligned}
$$


The system will be completed by adding to conjugation conditions $6.3-6.4$ and internal equations from 5.3 for all $\Omega_{i}$ :

$$
\mathbf{L}_{i}^{k}\left(U_{i}^{k+1}-U_{i}^{k}\right)-\mathbf{L}_{i}^{k-1}\left(U_{i}^{k}-U_{i}^{k-1}\right)+\omega^{2}\left(\widehat{\mathbf{L}}_{i}^{k}\right)^{-1} U_{i}^{k}=0, \quad U_{i}^{m+1}=0,
$$

where $k=2,3, \ldots, m$.

Replacing $\omega^{2}$ by $-\frac{d^{2}}{d t^{2}}$ we transform $6.4-6.5$ to the semi-discrete time-domain system (using the same notation $U_{i}^{k}$ for the time-domain solution)

$$
\begin{gathered}
\mathbf{L}_{i}^{k}\left(U_{i}^{k+1}-U_{i}^{k}\right)-\mathbf{L}_{i}^{k-1}\left(U_{i}^{k}-U_{i}^{k-1}\right)-\frac{d^{2}}{d t^{2}}\left(\widehat{\mathbf{L}}_{i}^{k}\right)^{-1} U_{i}^{k}=0, \\
U_{i}^{m+1}=0, \quad k=2,3, \ldots, m, \\
\widetilde{\mathbf{P}}_{i j}^{T}\left(\mathbf{L}_{i}^{1}\left(U_{i}^{2}-U_{i}^{1}\right)\right)+\widetilde{\mathbf{P}}_{j i}^{T}\left(\mathbf{L}_{j}^{1}\left(U_{j}^{2}-U_{j}^{1}\right)\right)- \\
\frac{d^{2}}{d t^{2}}\left(\widetilde{\mathbf{P}}_{i j}^{T}\left(\widehat{\mathbf{L}}_{i}^{1}\right)^{-1} U_{i}^{1}+\widetilde{\mathbf{P}}_{j i}^{T}\left(\widehat{\mathbf{L}}_{j}^{1}\right)^{-1} U_{j}^{1}\right)=\delta(t) \widetilde{g}_{i j},
\end{gathered}
$$

coupled with conjugation condition 6.3 and initial conditions

$$
\left.U_{i}^{1}\right|_{t<0}=0
$$

for $i=1, \ldots, N_{c}$ and for all $j \neq i$ such that $\Gamma_{i j} \neq \emptyset$.

ExAMPLE 5. In $1 D$ Example 1, the discretization (6.6), (6.7), (6.3) corresponds to the conventional finite-difference scheme on the spectrally-matched grid

$$
\frac{1}{\widehat{h}^{k}}\left(\frac{U^{k+1}-U^{k}}{h^{k}}-\frac{U^{k}-U^{k-1}}{h^{k-1}}\right)+\frac{d^{2}}{d t^{2}} U^{k}=g_{0} \delta_{k 0}, \quad k=-n+1, \ldots, n-1,
$$

with $U^{-n}=U^{n}=0$. Here $\delta$ is Kronecker's delta, split ROM solution $U$ is indexed as $U_{1}^{-k}=U^{k-1}, k=-n, \ldots,-1$ and $U_{2}^{k}=U^{k-1}, k=1, \ldots, n$, and the grid steps $\left\{h^{k}\right\}_{k=-n}^{n-1},\left\{\widehat{h}^{k}\right\}_{k=-n+1}^{n-1}$ are defined as

$$
\begin{aligned}
& h^{k}=\left(\mathbf{L}_{1}^{-k}\right)^{-1}, \quad k=-n, \ldots,-1, \\
& h^{k}=\left(\mathbf{L}_{2}^{k+1}\right)^{-1}, \quad k=0, \ldots, n-1, \\
& \widehat{h}^{k}=\left(\widehat{\mathbf{L}}_{1}^{-k+1}\right)^{-1}, \quad k=-n+1, \ldots,-1, \\
& \widehat{h}^{k}=\left(\widehat{\mathbf{L}}_{2}^{k+1}\right)^{-1}, \quad k=1, \ldots, n-1, \\
& \widehat{h}^{0}=\left(\widehat{\mathbf{L}}_{1}^{1}+\widehat{\mathbf{L}}_{2}^{1}\right)^{-1} .
\end{aligned}
$$

In Figure 6.1 we show an example of a $1 D$ spectrally-matched grid. In particular, we used ROMs of size 7 and 11 for the coarse grid cells in $\Omega_{1}=\{k \mid-n \leq k \leq 0\}$ and $\Omega_{2}=\{k \mid 0 \leq k \leq n\}$, respectively. We refer to $\left\{h^{k}\right\}_{k=-n}^{n-1}$ as the primary grid nodes and $\left\{\widehat{h}^{k}\right\}_{k=-n+1}^{n-1}$ as the dual grid nodes. Solution $U^{k}$ is defined at the primary grid, while the derivative $\left(U^{k+1}-U^{k}\right) / h^{k}$ is defined at the dual grid nodes.

REMARK 4. Note that in general equations (6.7) define a time-stepping scheme for the boundary solutions $U^{1}$ with a global mass matrix. The exception here is when matrices $\widehat{\mathbf{L}}_{i}^{1}$ are block diagonal such that their non-zero elements are $\widetilde{\mathbf{P}}_{i j}^{T} \widehat{\mathbf{L}}_{i}^{1} \widetilde{\mathbf{P}}_{i j}, j \in$ 


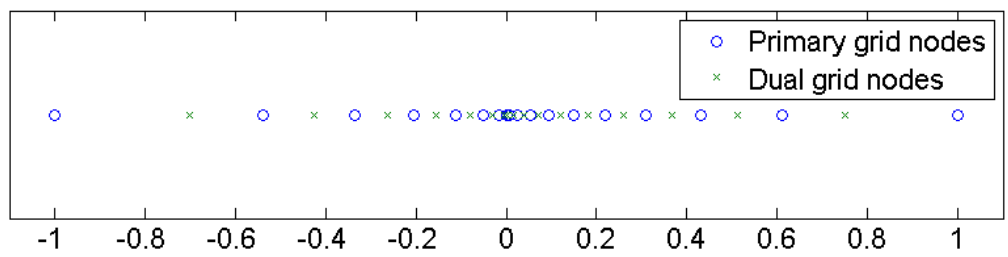

FIG. 6.1. A $1 D$ spectrally-matched grid. Blue circles are the primary (solution) grid nodes, green crosses are the dual (derivative) grid nodes.

$\mathcal{J}(i)$ only. This can be achieved by adding $\mathbf{P}_{i}^{\Gamma} \mathbf{S}_{i}$ to the projection subspace. Then for the solution at the shared boundary $\widetilde{u}_{i j}=\widetilde{u}_{j i}=\widetilde{\mathbf{P}}_{i j} U_{i}^{1}=\widetilde{\mathbf{P}}_{j i} U_{j}^{1}$ equation $(6.7)$ is replaced by

$$
\begin{aligned}
\frac{d^{2} \widetilde{u}_{i j}}{d t^{2}}= & \left(\left(\widetilde{\mathbf{P}}_{i j}^{T} \widehat{\mathbf{L}}_{i}^{1} \widetilde{\mathbf{P}}_{i j}\right)^{-1}+\left(\widetilde{\mathbf{P}}_{j i}^{T} \widehat{\mathbf{L}}_{j}^{1} \widetilde{\mathbf{P}}_{j i}\right)^{-1}\right)^{-1} \times \\
& \times\left(\widetilde{\mathbf{P}}_{j i} \mathbf{L}_{j}^{1}\left(U_{j}^{2}-U_{j}^{1}\right)+\widetilde{\mathbf{P}}_{i j} \mathbf{L}_{i}^{1}\left(U_{i}^{2}-U_{i}^{1}\right)\right)+\delta(t) \widetilde{g}_{i j},
\end{aligned}
$$

which only requires communication between the adjacent subdomains. Mass-matrix diagonalization by adding new elements to the projection subspace cannot increase the approximation error, in fact, it slightly improves accuracy. However, it may tighten the CFL stability limitation for the explicit time-stepping, which anyway will be still looser compared to the original problem (1.1), see remark 7 . Any standard time stepping scheme can be used for (6.6), (6.9) including Runge-Kutta, etc. The expressions on the right hand side of (6.6), (6.9) are always evaluated at the current time step.

REMARK 5. Every equation (6.3), (6.6), (6.9) contains at most three vector unknowns $U_{i}^{j}$ and $\widetilde{u}_{i j}$, that can be interpreted as boundary restrictions of the solution, see Remark 3. Schematically the stencil of the obtained operator is shown in Fig. 6.2. So, in general the structure of (6.3), (6.6), (6.9) mimics the matrix structure of the second-order finite-volume discretization schemes.

7. Method summary. We summarize below our method as an algorithm that is well suited for parallel HPC platforms.

Algorithm 1 (Multiscale S-fraction ROM method).

Stage 1 (off-line). In full parallel mode for each subdomain $\Omega_{i}$ do the following:

(1.1) Compute the projection subspace basis $\mathbf{V}_{i}$ and the the projected operators $\mathbf{A}_{i}^{m}$, $\mathbf{B}_{i}^{m}, \mathbf{S}_{i}^{m}$ that define the ROM (4.6).

(1.2) Apply the block Lanczos algorithm with an inner product $\mathbf{B}_{i}^{m}$ to transform $\left(\mathbf{A}_{i}^{m}, \mathbf{S}_{i}^{m}\right)$ to a block tridiagonal form $\left(\mathbf{T}_{i}, \mathbf{R}_{i}\right)$.

(1.3) Obtain the S-fraction coefficients $\mathbf{L}_{i}^{k}, \widehat{\mathbf{L}}_{i}^{k}$ from $\left(\mathbf{T}_{i}, \mathbf{R}_{i}\right)$ using relations C.5) 田.

(1.4) Obtain $\widetilde{g}_{i j}$ and $\widetilde{q}_{i j}$ by projecting $\left.g\right|_{\Gamma_{i j}}$ and $\left.q\right|_{\Gamma_{i j}}$ onto $\mathbf{S}_{i j}$, respectively.

Stage 2 (on-line). Starting with initial conditions $\left.U_{i}^{k}\right|_{t=0}=\left.\partial_{t} U_{i}^{k}\right|_{t=0}=0$ for each time step do the following:

(2.1) Exchange $\widetilde{\mathbf{P}}_{i j} \mathbf{L}_{i}^{1}\left(U_{i}^{2}-U_{i}^{1}\right)$ and $\widetilde{\mathbf{P}}_{j i} \mathbf{L}_{j}^{1}\left(U_{j}^{2}-U_{j}^{1}\right)$ between the subdomains sharing $\Gamma_{i j}$.

(2.2) While waiting for the data exchange, compute in parallel for each $\Omega_{i}$ the updates to the interior solutions $U_{i}^{2}, \ldots, U_{i}^{m}$ using (6.6).

\footnotetext{
${ }^{\dagger}$ Steps (1.2) and (1.3) can be combined using a particular form of block Lanczos method.
} 


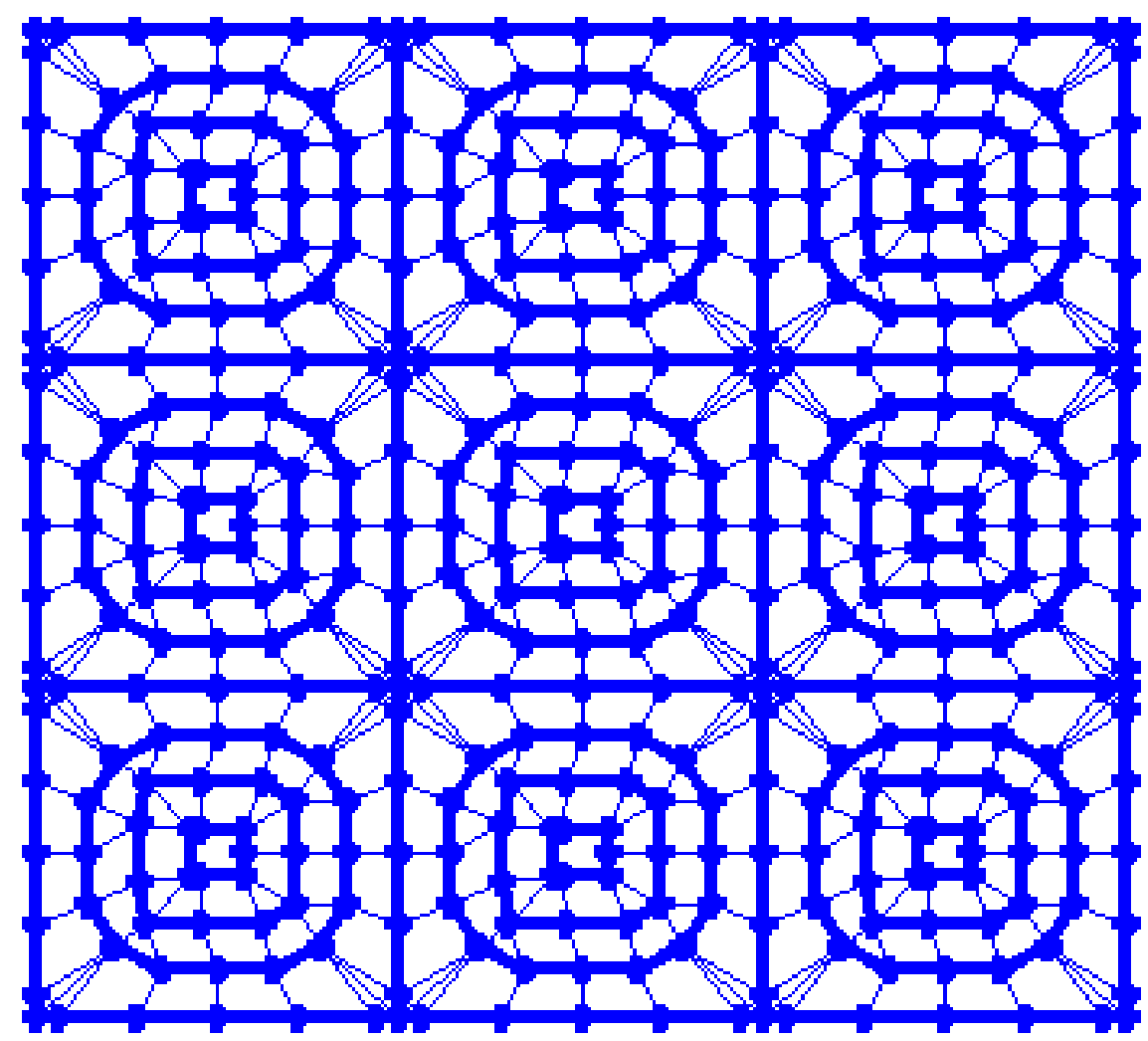

FIG. 6.2. The stencil of the $S$-fraction multiscale ROM of $\mathbf{A}$ with $3 \times 3$ coarse cells. Adjacent subdomains communicate through the boundary unknowns only, and interior unknowns are arranged in hidden layers. This is schematic representation similar to the one in the Figure [5.1. See the caption thereof.

(2.3) Once the data exchange is complete, compute in parallel for each $\Omega_{i}$ the updates to the boundary solutions $U_{i}^{1}$ using 6.9.). Use 6.2) to compute the solution at receivers.

The main computational cost of the online part in vector to matrix multiplications in $(2.2-2.3)$ is $O\left(\widetilde{K}_{i}^{2} m_{i}\right)$ per coarse cell per step, i.e., it grows as power $5 / 3$ of the total degrees of freedom (i.e., McMillan degree) in the coarse cell (assuming consistent discretization with $\widetilde{K}_{i}=O\left(m_{i}^{2}\right)$ ). Note that due to block-tridiagonal structure of 6.6 step (2.2) can be efficiently palatalized further reducing the cost. Also, the order of steps (2.1) and (2.2) allows for what is known in computer science literature as hiding the communication latency behind the computations. We should point out, that the communication cost $O\left(\widetilde{K}_{i}\right)$ per step per cell is very low. We only exchange projected shared boundary degrees of freedom between the adjacent subdomains as if we had a second order scheme, even though in our case this number is much smaller. Such a small communication cost is possible because the ROMs approximate the NtD map to high (spectral) accuracy, even though (5.2)-(5.3) resemble a three-point difference scheme.

The number of boundary basis functions $\widetilde{K}_{i}$ grows with the number of the minimal wavelengths $L$ in the coarse cells. The dimensionality reduction factor $\frac{N_{i}}{\widetilde{K}_{i} m_{i}}$ 
also grows with $L$. So the optimal $L$ will be the maximum of two values: (i) the breakeven point between $O\left(\widetilde{K}_{i}^{2} m_{i}\right)$ computational and $O\left(\widetilde{K}_{i}\right)$ communication cost; (ii) the optimal point of total computational cost in the entire computational domain, that can be obviously estimated as $O\left(\frac{\widetilde{K}_{i}^{2} m_{i}}{L^{3}}\right)$ for the $3 \mathrm{D}$ (plus one dimension of time) wave problems. The reduction is limited by the Nyquist sampling rate (two degrees of freedom per wavelength), so there is no significant benefits to further increase $L$ when the ROM approaches that limit.

REMARK 6. Recall (see Figure 3.1) that we consider graphs with the corner nodes removed. We assumed that such a modification adds an insignificant error, however this error can be corrected by equating values $\left\{\widetilde{u}_{l}\right\}_{l \in \mathcal{H}(k)}$ at the hanging nodes corresponding to the corner node $k \in \Gamma^{\cap}$. The cost of the correction algorithm (described in the Appendix (D) is insignificant compared to Algorithm 1 in both computation and communication.

8. Stieltjesness of the multiscale reduced order transfer function. Let $\mathbf{P}_{\Gamma}$ be the prolongation operator from domain $\Omega$ to the partition skeleton $\Gamma$ (i.e., $\mathbf{P}_{\Gamma}^{T}$ is the projection from $\Omega$ to $\Gamma$ ). Assuming the "no corners" condition (3.8), we define the skeleton transfer function as

$$
\mathbf{F}\left(\omega^{2}\right)=\mathbf{P}_{\Gamma}^{T}\left(\mathbf{A}+\omega^{2} \mathbf{B}\right)^{-1} \mathbf{P}_{\Gamma}^{T} \in \mathbb{C}^{K \times K},
$$

where $K$ is the number of nodes in the partition skeleton $\Gamma, K=\sum_{i, j} K_{i j}$. It maps from inputs to outputs satisfying condition 2.2 , i.e.,

$$
\left.\mathbf{F}\left(\omega^{2}\right) g\right|_{\Gamma}=u_{\Gamma}
$$

Following the same reasoning as for $\mathbf{F}_{i}$ outlined in Remark 1 $\mathbf{F}$ is the Stieltjes matrix valued function of $\omega^{2}$, i.e. it can be represented as

$$
\mathbf{F}\left(\omega^{2}\right)=\sum_{l=1}^{N} \frac{v_{l} v_{l}^{T}}{\lambda_{l}+\omega^{2}},
$$

were $v_{l}=\mathbf{P}_{\Gamma}^{T} z_{l} \in \mathbb{R}^{K}$. It is the Stieltjes matrix-valued function of order $K$ according to Definition 1

Our multiscale reduced order model in the frequency domain can be viewed as an approximation to $\mathbf{F}\left(\omega^{2}\right)$ defined as follows (assuming steps 1-3 are performed for all adjacent $\Omega_{i}, \Omega_{j}$ ).

Algorithm 2.

1. Given input $\left.g\right|_{\Gamma}$ compute $\widetilde{g}_{i j}=\left.\mathbf{S}_{i j}^{T} g\right|_{\Gamma_{i j}}$.

2. Solve (6.4), 6.5) and (6.3) in terms of $\widetilde{u}_{i j}$.

3. Compute $\left.u\right|_{\Gamma_{i j}} \approx \mathbf{S}_{i j} \widetilde{u}_{i j}$.

The following results show that the multiscale reduced order model given by $\mathrm{Al}$ gorirthm 2 is uniquely defined under Assumption 2 for Laplace frequencies satisfying conditions 3.2 and its time-domain realization in Algorithm 1 is stable and conservative.

Let $\widetilde{K}=\sum_{i, j} \widetilde{K}_{i j}, \widetilde{g} \in \mathbb{R}^{\widetilde{K}}$ and $\widetilde{u} \in \mathbb{R}^{\widetilde{K}}$ are the direct sums of respectively $\widetilde{g}_{i j}$ and $\widetilde{u}_{i j}$ for all adjacent $\Omega_{i}$ and $\Omega_{j}$. We define $\widetilde{\mathbf{F}}^{m}(\lambda): \mathbb{C} \rightarrow \mathbb{C}^{\widetilde{K} \times \widetilde{K}}$ via a linear map

$$
\widetilde{\mathbf{F}}^{m}(\lambda) \widetilde{g}=\widetilde{u}
$$


Proposition 8.1. If Assumption 2 is valid for all subdomains, then map $\widetilde{\mathbf{F}}^{m}(\lambda)$ is $(0, \infty)$-Stieltjes function of order $\widetilde{K}$. Moreover, the non-negative definiteness can be replaced by positive-definiteness in the conditions 2 and 3 of Definition 1.

Proof. Direct calculation yields

$$
\widetilde{\mathbf{F}}^{m}(\lambda)^{-1}=\sum_{i=1}^{N_{c}} \mathbf{P}_{\Gamma}^{\Gamma_{i}}\left(\widetilde{\mathbf{F}}_{i}^{m}(\lambda)\right)^{-1} \mathbf{P}_{\Gamma}^{\Gamma_{i}^{T}},
$$

where the conjugate to $\mathbf{P}_{\Gamma}^{\Gamma_{i}} \in \mathbb{R}^{\widetilde{K} \times \widetilde{K}_{i}}$ is the projection operator from the space of compressed inputs (outputs) supported on $\Gamma$ to the ones supported on $\Gamma_{i}$. Substituting in place of $\left(\widetilde{\mathbf{F}}_{i}^{m}(\lambda)\right)^{-1}$ its representation via the S-fraction 5.1 we obtain

$$
\widetilde{\mathbf{F}}^{m}(\lambda)^{-1}=\sum_{i=1}^{N_{c}} \mathbf{P}_{\Gamma}^{\Gamma_{i}} \mathbf{S}_{i}\left(-\frac{\lambda}{\widehat{\mathbf{L}}_{i}^{1}}+\frac{1}{\frac{1}{\mathbf{L}_{i}^{1}}+\frac{1}{\ddots+\frac{1}{-\frac{\lambda}{\widehat{\mathbf{L}}_{i}^{m}}+\mathbf{L}_{i}^{m}}}}\right) \mathbf{S}_{i}^{T} \mathbf{P}_{\Gamma}^{\Gamma_{i}{ }^{T}},
$$

where $\mathbf{L}_{i}^{k}, \widehat{\mathbf{L}}_{i}^{k}$ are the coefficients of 5.1 at $\Omega_{i}$. Since these matrices are symmetric positive-definite, the matrices in the brackets in (8.1) are symmetric positive-definite for $\lambda \in(-\infty, 0)$ and their imaginary parts are symmetric-positive definite for $\Im \lambda>0$. Then the same holds for $\left(\widetilde{\mathbf{F}}^{m}(\lambda)\right)^{-1}$ (thanks to non-singularity of $\sum_{i=1}^{N_{c}} \mathbf{P}_{\Gamma}^{\Gamma_{i}} \mathbf{P}_{\Gamma}^{\Gamma_{i}{ }^{T}}$ ) and obviously the same is true for $\widetilde{\mathbf{F}}^{m}(\lambda)$.

REMARK 7. The Stieltjes property implies stability and energy conservation of the reduced order model [1]. The strong Stieltjes property of reduced order transfer function stated in Proposition 8.1 is intrinsic for Galerkin-type approximations of symmetric nonpositive operator pencil problems. In fact, it can be shown, that $\widetilde{F}$ can be equivalently obtained via the multi-scale Galerkin spectral element method using the basis of block-rational Krylov subspaces (4.5) in every subdomain, e.g., see [12] for the Galerkin-equivalence result in the $1 D$ framework. The CFL limiting time-step for the Galerkin approximation can not be smaller than the one for the exact (fine grid) problem, as confirmed numerically for our multiscale ROM in the following section. By exploiting this connection it is also possible to lift condition (2.2) (at the expense of some convergence speed). The rigorous analysis of the Galerkin connection is in progress

9. Numerical examples. We first illustrate the performance of our approach on an example of a scalar wave equation

$$
\nabla \cdot(\sigma \nabla u)-u_{t t}=g \delta^{\prime}(t)
$$

in a $3 \mathrm{D}$ cube $[0 ; 4]^{3} \mathrm{~km}^{3}$ with homogeneous Dirichlet boundary conditions. In all the experiments we considered $g$ supported and smoothly varied on the boundary of subdomains. Two scenarios were considered: homogeneous medium with $\sigma=$ $1 \mathrm{~km}^{2} / \mathrm{s}^{2}$ and a heterogeneous case shown in Figure 9.1. For both cases, source and receiver were centered at $(1 ; 1.5 ; 1.5)$ and $(3 ; 2.5 ; 2.5)$, respectively. The operator $\mathbf{A}$ 
in (1.1) was obtained a from second-order discretization of 9.1) on the equidistant reference grid with $160 \times 160 \times 160$ nodes. The signal was convolved then with Gaussian wavelet with cutoff frequency about $2 \pi s^{-1}$, and, consequently, the corresponding minimum wavelength is about $1 \mathrm{~km}$. We split the entire grid into $4 \times 4 \times 4=64$ equal cubic subdomains $\Omega_{i}$ (see a 2D slice in Figure 9.1 on the right), so their edges have a length about a minimum wavelength. In all our experiments for all subdomains we used the same number of $m_{i}=m$ in $\mathcal{V}_{m_{i}}$ (equivalently, ROM layers) and the same number of boundary solutions $\widetilde{K}_{i}=\widetilde{K}$.
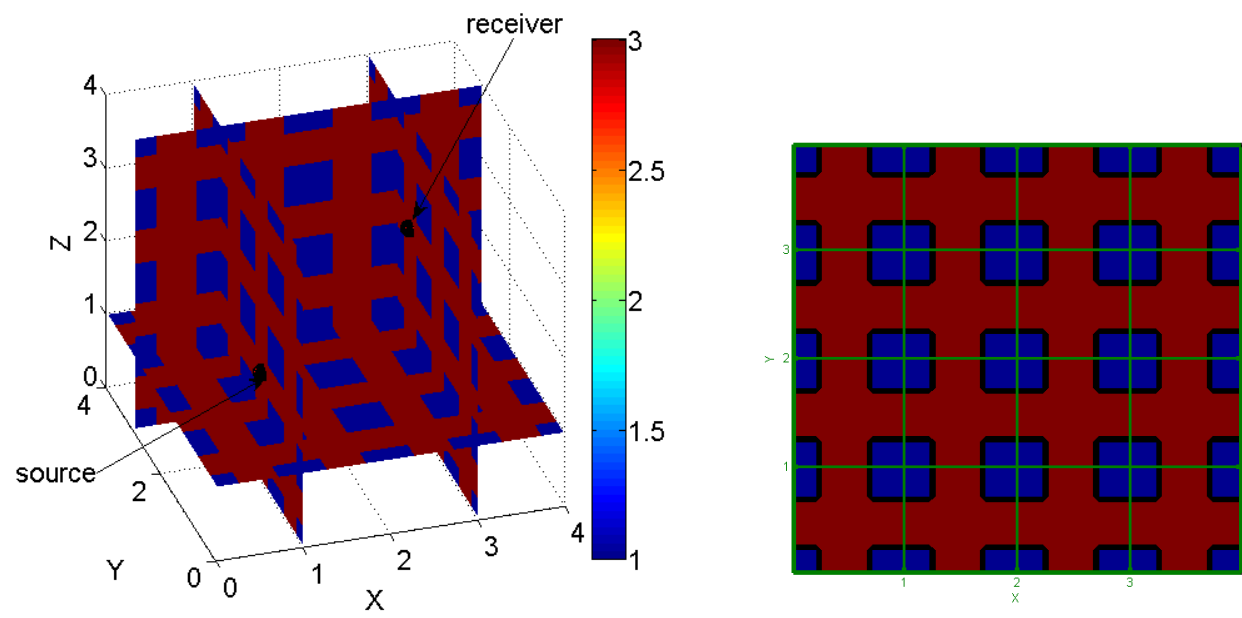

FIG. 9.1. Heterogeneous medium for the acoustic problem 9.1) (left) and a slice of its splitting into subdomains shown by green lines (right).

We start with benchmarking our MSSFROM algorithm against fine-scale solution for both scenarios. For multi-scale solver we used parameters $m=4$ and $\widetilde{K}=180$. The results of simulations are shown in Figure 9.2. For the homogeneous case, serial computation times were 0.2 hours and 2.2 hours for the multi-scale and full-scale solvers, respectively, and CFL numbers were approximately the same. For the heterogeneous scenario, the serial computation time for the multi-scale solver remained the same 0.2 hours, while the full-scale solver required 3.3 hours. That is caused by the difference in CFL numbers: for the full-scale solver it was lower by a factor of 1.5 .
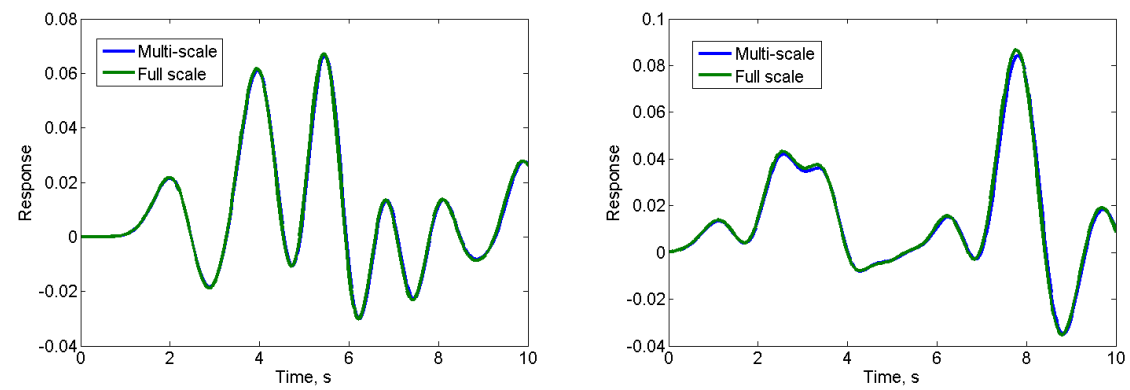

FIG. 9.2. Simulations results using MSSFROM and full-scale solver for homegeneous (left) and heterogenous (right) media 
In our next example we perform the numerical dispersion study of MSSFROM. For homogenous case scenario with unit speed $\sigma=1$, we fixed parameters $m=4$ and $K=150$ and changed the cut-off frequency $\omega_{\max }$. We note that, while the size of subspace remained the same, the shape of the boundary basis functions (and, consequently, the subspace $\mathcal{V}_{m}$ ) depended on the frequency range under consideration (see equation 4.4). The number of degrees of freedom used for approximating the solution at each coarse cell is $m K$. Hence, assuming the coarse cell edge is of unit length, $\frac{2 \pi \sigma \sqrt[3]{m K}}{\omega_{\max }}$ degrees of freedom (ppw) were used per wavelength. In Figure 9.3 we plot the error of MSSFROM with respect to the true fine-scale solution without corner set removal. As one can observe, MSSFROM provides good accuracy up to about 4 ppw.

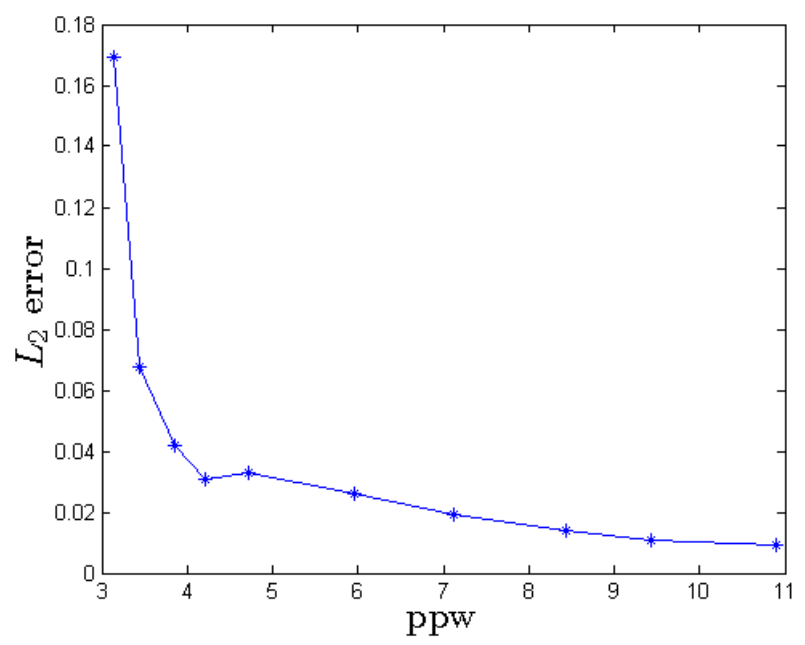

FIG. 9.3. $L_{2}$ error with respect to the number of degrees of freedom per wavelength. For coarse cell edge of unit length that number can be estimated as $\frac{2 \pi \sigma \sqrt[3]{m K}}{\omega_{\max }}$.

Next, we perform the convergence study of the MSSFROM approach. In the first set of experiments we kept the subspace of boundary solutions fixed with $\widetilde{K}=294$ and increased $m$. In Figure 9.4 we show the maximum absolute error for different value of $m=2,3,4,5$. For both scenarios the algorithm demonstrates exponential convergence. Also, for the heterogeneous case $\sigma$ varies from 1 to 3 while for the homogenous one it is equal to 1 . Therefore, a smaller subspace is required to approximate the former case. This explains why the convergence is faster in the heterogeneous case compared to the homogeneous scenario.

In the next experiment we fixed $m=4$ and were increasing $K_{i}=K$ for all $i$ simultaneously. Since the length of each edge of $\Omega_{i}$ is about a minimum wavelength, $\sqrt{K / 6}$ shows how many degrees of freedom per wavelength are used for approximating boundary solutions. In Figure 9.5 we show the errors for the homogeneous and heterogeneous cases versus $\sqrt{K / 6}$. In both cases convergence is exponential, with plateaus while $\sqrt{K / 6}$ changes from one integer to the consequent one. We also note that the error for the heterogeneous case is worse than for the homogeneous one because of the formulation we used to compute boundary solutions. Indeed, for a given face of the subdomain we assumed that the medium is uniform in the direction normal to 


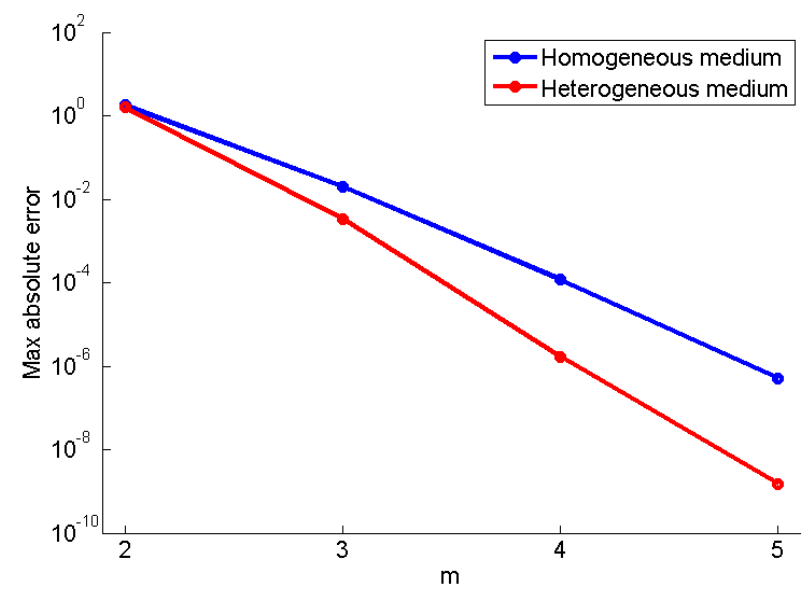

FIG. 9.4. Convergence curves with respect to $m$ for homogeneous and heterogeneous cases. While both show exponential convergence, the latter is faster because of inhomogeneity.

the face. For the heterogeneous case it slightly worsens the approximation properties of the boundary solutions subspace.

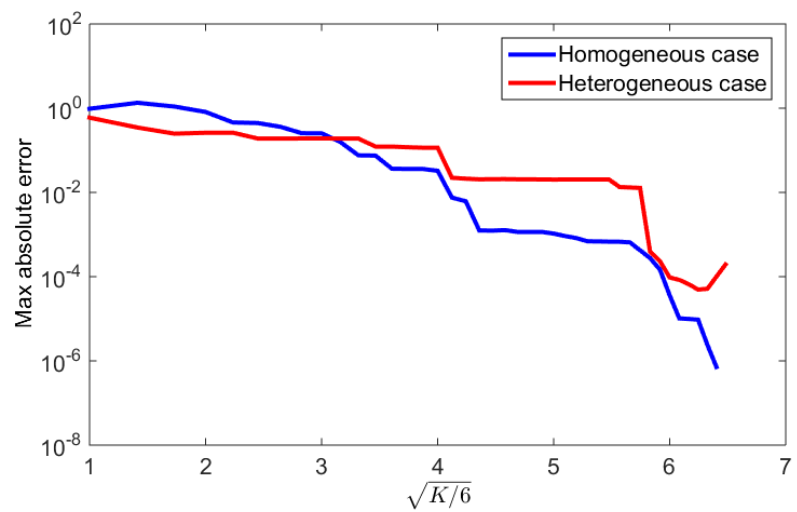

FIG. 9.5. Convergence curves for homogeneous and heterogeneous cases. Both scenarios show exponential convergence, with plateaus while $\sqrt{K / 6}$ changes from one integer to the next one. Here $\sqrt{K / 6}$ is approximately equal to the number of degrees of the freedom per wavelength for the coarse cell boundary discretization. Heterogeneous case converges slower because of the approximation we used to construct the boundary solutions.

In our next experiment we consider a 3D anisotropic elastodynamic problem

$$
\begin{array}{r}
u_{i, t t}=\sigma_{i j, j}, \\
\sigma_{i j}=C_{i j k l}\left(u_{k, l}+u_{l, k}\right) / 2
\end{array}
$$

in the cube $[0,5]^{3} \mathrm{~km}^{3}$ with homogeneous Dirichlet boundary conditions. Here $u_{i}$ and $\sigma_{i j}$ are components of displacement vector and stress tensor, respectively. $C_{i j k l}$ are elastic moduli tensor components. In isotropic medium it has a form $C_{i j k l}=$ $\lambda \delta_{i j} \delta_{k l}+\mu\left(\delta_{i k} \delta_{j l}+\delta_{i l} \delta_{j k}\right)$. The locations of $x$-oriented stress source receiver measuring 
$x$ - component of the displacement were $(1 ; 2.5 ; 2.5)$ and $(4 ; 2.5 ; 2.5)$, respectively. The medium (see Figure 9.6 consists of water-filled fracture and cavities (blue) with $\frac{\lambda}{\rho}=2 \frac{\mathrm{km}^{2}}{\mathrm{~s}^{2}}, \frac{\mu}{\rho}=0$ and empty air-filled cavity (white) with $\frac{\lambda}{\rho}=0, \frac{\mu}{\rho}=0$ embedded in slow isotropic background (cyan) with $\frac{\lambda}{\rho}=2 \frac{\mathrm{km}^{2}}{\mathrm{~s}^{2}}$ and $\frac{\mu}{\rho}=1 \frac{\mathrm{km}^{2}}{\mathrm{~s}^{2}}$. The background is surrounded by fast anisotropic orthorhombic (red) layers with non-zero components of elastic moduli tensor $\frac{C_{1111}}{\rho}=8 \frac{\mathrm{km}^{2}}{\mathrm{~s}^{2}}, \frac{C_{1122}}{\rho}=6 \frac{\mathrm{km}^{2}}{\mathrm{~s}^{2}}, \frac{C_{1133}}{\rho}=5 \frac{\mathrm{km}^{2}}{\mathrm{~s}^{2}}, \frac{C_{2222}}{\rho}=9 \frac{\mathrm{km}^{2}}{\mathrm{~s}^{2}}$, $\frac{C_{2233}}{\rho}=4 \frac{\mathrm{km}^{2}}{\mathrm{~s}^{2}}, \frac{C_{3333}}{\rho}=10 \frac{\mathrm{km}^{2}}{\mathrm{~s}^{2}}, \frac{C_{1212}}{\rho}=1 \frac{\mathrm{km}^{2}}{\mathrm{~s}^{2}}, \frac{C_{2323}}{\rho}=2 \frac{\mathrm{km}^{2}}{\mathrm{~s}^{2}}, \frac{C_{1313}}{\rho}=3 \frac{\mathrm{km}^{2}}{\mathrm{~s}^{2}}$. The minimum wavelength at cutoff frequency is approximately $1 \mathrm{~km}$. The fine reference grid with $200 \times 200 \times 200$ nodes has been split into $5 \times 5 \times 5=125$ subdomains. To construct the ROMs, we used $m=4$ and $K=75$ for all subdomains, i.e. we ended up with $6 \times 75 \times 4=1800$ degrees of freedom per subdomain instead of $40 \times 40 \times 40 \times 3=$ 192000 for the full-scale reference discretization.
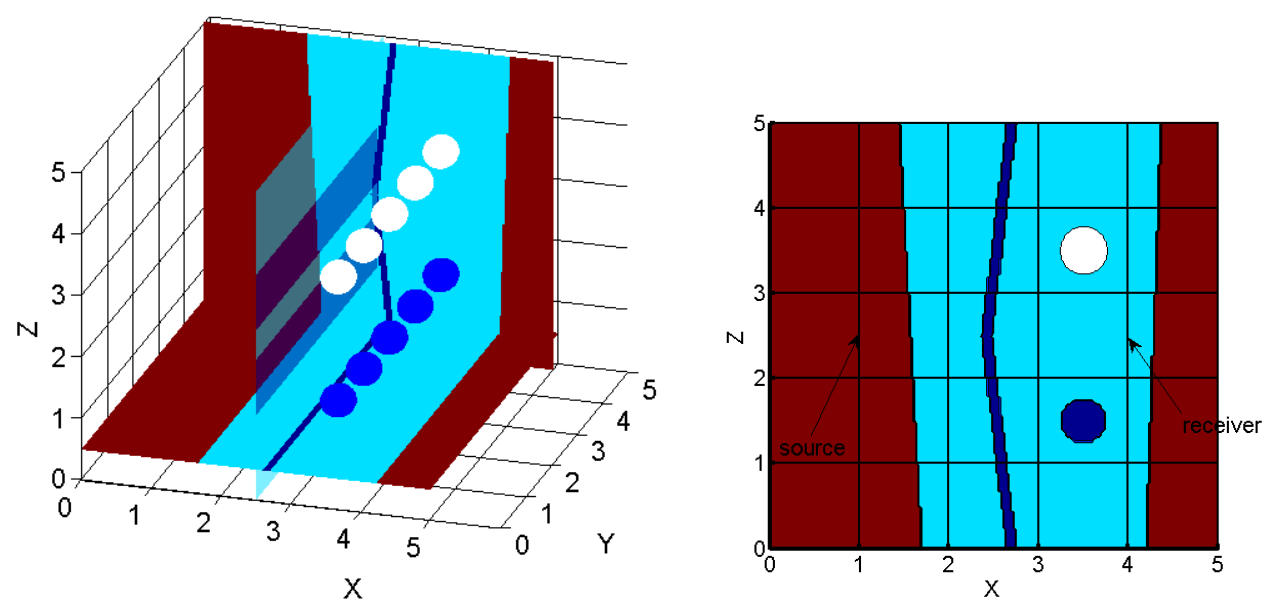

FIG. 9.6. $3 D$ medium (left) and its slice at $y=2.5 \mathrm{~km}$ for elastodynamic benchmark. The formation consists of slow isotropic (cyan) and fast anisotropic (red) parts with water-filled fracture and cavity (blue) and air-filled cavity (white).

A comparison of the solution at the receiver (see Figure 9.6 for source and receiver positions) between our multiscale method and the reference solution is given in Figure 9.7 Again, here we report the CPU times for serial implementations of both methods. Though the reduction in the number of unknowns was more than two orders of magnitude, the speed up in the simulation time (on-line part only vs the reference full-scale simulation) was less: 0.26 hour vs 10.5 hours. That is caused by the dense structure of matrices $\widehat{\mathbf{L}}^{k}$ and $\mathbf{L}^{k}$ which results in larger computational cost (per unknown) of the matrix multiplication in the time-stepping scheme 6.6, 6.9). We also note that CFL numbers for multi-scale and full-scale solvers were approximately the same in this example. Even though the speedup in the serial case is already substantial, the parallel implementation will favor our multiscale approach even more due to low ratio of communication and computation costs, as it was emphasized in Section 7. An efficient parallel implementation and testing is a topic of future work.

10. Conclusion. We developed a multi-scale reduced-order modeling approach for efficient large wavefield simulations. Similar to the conventional multi-scale meth- 


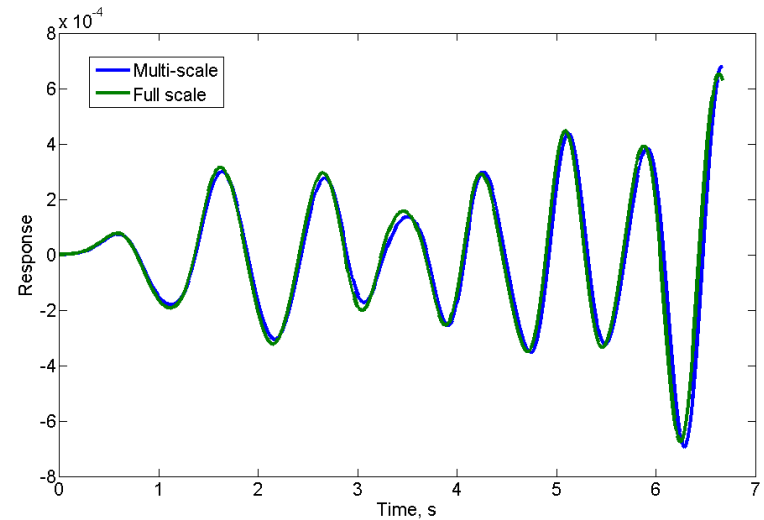

FIG. 9.7. 3D anisotropic elastodynamic problem. Multi-scale solver against full-scale solver.

ods, our algorithm consists of the off-line and the on-line stages. At the first stage a reduced-order multi-scale model is constructed. In particular, we start with the fine grid discretization of the spatial operator and split the grid into subdomains (coarse cells). The divide-and-conquer-type algorithm was suggested to construct the corresponding partitioning of the operator. Then we reduce the number of degrees of freedom in the partitioning by substituting it with a high-accuracy reduced-order model. The crucial step of our approach is the transformation of the reduced-order model to S-fraction form. This results in the sparsified (block-tridiagonal) stencil of the obtained discrete spatial operator, reduction of communications between the subdomains and greatly reduced overall computational cost. The off-line stage is embarrassingly parallel and is performed just once for the entire time-domain simulations and for all sources. At the on-line stage, the time-domain simulations are performed within the obtained multi-scale ROM formulation. The significantly reduced number of unknowns and the sparsified stencil results in a speed up of our approach by a factor of up to 30 compared to full-scale simulations even on serial computer. Due to the reduced communications between subdomains, we expect even more dramatic results when an MPI and/or GPU implementation is ready.

Besides the high performance computing implementation, there remains a number of open algorithmic and theoretical problems, successful resolution of which may lead to a better understanding of the developed approach and its further improvements. Some of these issues are listed below.

- Removal of the restriction on inputs and outputs to be supported only at the boundary of course cells. That can be done by establishing a connection between our approach and conventional multiscale and spectral element methods.

- Making the Courant-Friedrichs-Lewy (CFL) stability condition consistent with the Nyquist limit corresponding to the source frequency bandwidth.

- Obtaining compact representation of matrix S-fraction coefficients, and as result, further decrease the computational cost of the time-stepping (online stage).

11. Acknowledgments. We are grateful to Smaine Zeroug for bringing our attention to the large scale modeling in seismic exploration, Leonid Knizhnerman and 
Yousef Saad for their invaluable contribution to the POD algorithm for the construction of boundary subspaces [8] and also Alexander Aptekarev and Yuri Dyukarev for introducing work [13.

\section{Appendix A. Divide and conquer domain partitioning.}

A.1. Two subdomains. The adjacent nodes satisfy the following symmetry property that follows straightforwardly from symmetry of $\mathbf{A}$.

Lemma A.1. If $\mathcal{A}\left(\dot{\Omega}^{\prime}\right) \cap \AA^{\prime \prime}=\emptyset$ then $\mathcal{A}\left(\dot{\Omega}^{\prime \prime}\right) \cap \AA^{\prime}=\emptyset$. Also, since the diagonal entries of $\mathbf{A}$ are assumed to be all non-zero, we have $\Omega^{\prime} \subset \mathcal{A}\left(\AA^{\prime}\right)$ for any $\Omega^{\prime}$.

Proposition 1. Let there exist a nonempty $\Omega \subsetneq \Omega$, such that $\mathcal{A}(\Omega) \subsetneq \Omega$. Then there exist disjoint subdomains $\Omega_{1}, \Omega_{2} \neq \emptyset$ and $\Gamma$ satisfying conditions

$$
\Omega=\AA_{1} \cup \AA_{2} \cup \Gamma \text {, and } \Gamma=\mathcal{A}\left(\AA_{1}\right) \backslash \AA_{1}=\mathcal{A}\left(\AA_{2}\right) \backslash \AA_{2} .
$$

Proof. The proof is constructive. We choose $\AA_{2}=\Omega \backslash \mathcal{A}(\Omega), \AA_{1}=\Omega \backslash \mathcal{A}\left(\AA_{2}\right)$, $\Gamma=\Omega \backslash \check{\Omega}_{1} \backslash \AA_{2}$. By construction $\AA_{1}, \AA_{2} \neq \emptyset$, all three subdomains are disjoint and $\Omega$ is their union. Then

$$
\Gamma=\Omega \backslash \stackrel{\Omega}{\Omega}_{1} \backslash \stackrel{\Omega}{\Omega}_{2}=\Omega \backslash\left(\Omega \backslash \mathcal{A}\left(\stackrel{\Omega}{\Omega}_{2}\right)\right) \backslash \stackrel{\Omega}{2}_{2}=\mathcal{A}\left(\stackrel{\Omega}{2}_{2}\right) \backslash \stackrel{\Omega}{2}_{2} .
$$

By construction, $\stackrel{\Omega}{\Omega}_{1}$ is a complement to $\mathcal{A}\left(\AA_{2}\right)$, so from Lemma A.1 we conclude, that $\mathcal{A}\left(\Omega_{1}\right) \cap \Omega_{2}=\emptyset$. Hence, $\mathcal{A}\left(\Omega_{1}\right) \backslash \AA_{1} \subset \Gamma$ and, to show that $\Gamma=\mathcal{A}\left(\Omega_{1}\right) \backslash \Omega_{1}$, it remains to prove that all elements of $\Gamma$ are in $\mathcal{A}\left(\Omega_{1}\right)$. Again, from the definition of $\Omega_{2}$ and Lemma A.1 it follows that $\Omega \cap \mathcal{A}\left(\Omega_{2}\right)=\emptyset$ and, consequently, $\Gamma \cap \Omega=\emptyset$. Therefore, since $\Gamma=\mathcal{A}(\stackrel{\Omega}{)}) \backslash \Omega_{1}$, we have $\Omega_{1} \supseteq \Omega$ and, obviously, $\mathcal{A}\left(\Omega_{1}\right) \supseteq \mathcal{A}(\Omega)$. By construction $\Gamma \subset \mathcal{A}(\Omega)$, so $\Gamma \subset \mathcal{A}\left(\Omega_{1}\right)$.

Obviously, any $\mathbf{A}$ with at least one trivial non-diagonal element satisfies the condition of Proposition 1 . The case $\Gamma=\emptyset$ yields decoupled problems on $\AA_{1}$ and $\check{\Omega}_{2}$. The proof of the Proposition 1 provides an algorithm for partitioning of a sparse matrix A. We denote by $\Omega_{i}=\Omega_{i} \cup \Gamma$ of size (number of nodes) $N_{i}$ and by $\mathbf{A}^{i} \in \mathbb{R}^{N_{i} \times N_{i}}$ (with elements $a_{k l}^{i}$ ) the split operators defined on the nodes of $\Omega_{i}, i=1,2$ defined via the following three step algorithm.

Algorithm 3. According to Proposition 1, $a_{k l}=0$ if $k$ and $l$ belong to different $\stackrel{\Omega}{\Omega}_{i}$, so such elements are excluded.

1. For all pairs of indices $(k, l)$, such that at least one of them belongs to $\AA_{i}$, $i=1,2$ set

$$
a_{k l}^{i}=a_{k l} .
$$

2. For all $k \neq l$, such that $k, l \in \Gamma$, set

$$
a_{k l}^{1}=\alpha_{k l} a_{k l}, \quad a_{k l}^{2}=\left(1-\alpha_{k l}\right) a_{k l},
$$

where $0 \leq \alpha_{k l}=\alpha_{l k} \leq 1$.

3. For $k \in \Gamma$ set $a_{k k}^{i}=a_{k k} \frac{s_{i}}{s_{1}+s_{2}}, i=1,2$, where

$$
s_{i}=\sum_{l \neq k}\left|a_{k l}^{i}\right|
$$


For the splitting of 3.1 we also need the splitting of $\mathbf{B}^{i}=\operatorname{diag}\left\{b_{k}^{i}\right\}$ via a simplified version of Algorithm 3 performed as follows

$$
b_{k}^{i}= \begin{cases}b_{k}, & \text { if } k \in \stackrel{\Omega}{\Omega}_{i}, \\ \left((i-1)+(-1)^{i+1} \beta_{k}\right) b_{k}, & \text { otherwise. }\end{cases}
$$

where $0<\beta_{k}<1$ and $i=1,2$. Normally, $\alpha_{i j}$ and $\beta_{i}$ are chosen to approximately balance the condition numbers of partitioned problems.

By construction, matrices $\mathbf{A}^{i}, \mathbf{B}^{i}$ obtained by Algorithm 3 satisfy the identity

$$
\mathbf{A}+\omega^{2} \mathbf{B}=\sum_{i=1}^{2} \mathbf{P}_{i}\left(\mathbf{A}^{i}+\omega^{2} \mathbf{B}^{i}\right) \mathbf{P}_{i}^{T}, \quad \forall \omega^{2} \in \mathbb{C} .
$$

For the stability of the reduced order model it is important that $\mathbf{A}^{i}$ inherit the non-positive definiteness of $\mathbf{A}$. The following proposition shows an even stronger result for the important class of diagonally dominant matrices arising from discretization of second order elliptic operators.

Proposition 2. Let $\mathbf{A}$ be diagonally dominant, i.e., $\forall k\left|a_{k k}\right| \geq \sum_{l \neq k}\left|a_{k l}\right|$. Then $\mathbf{A}^{i}, i=1,2$ obtained via Algorithm 3 are also diagonally dominant non-positive definite matrices.

Proof. According to Proposition 1 , if $l \in \Omega_{i}$ then $\forall k a_{k l}^{i}=a_{k l}$. For the rows with diagonals $k$ in $\Gamma$, from the dominance of the corresponding row of $\mathbf{A}$ and from Algorithm 3 we obtain diagonal dominance

$$
\sum_{l \neq k}\left|a_{k l}^{i}\right|=\frac{s_{i}}{s_{1}+s_{2}} \sum_{l \neq k}\left|a_{k l}\right| \leq \frac{s_{i}}{s_{1}+s_{2}}\left|a_{k k}\right|=\left|a_{k k}^{i}\right| .
$$

From the non-positive definiteness and diagonal dominance of $\mathbf{A}$ its diagonal is negative and so are the diagonals of $\mathbf{A}^{i}$, that together with diagonal dominance of the latter yields their non-positive definiteness.

A.2. Multidomain algorithm. We employ Algorithm 3 as an elementary step of our "divide and conquer" graph partitioning algorithm presented below.

Algorithm 4.

Initialize the partition with $\Omega_{1}=\Omega, \mathbf{A}_{1}=\mathbf{A}, \mathbf{B}_{1}=\mathbf{B}$.

For $p=1,2, \ldots$ perform the following:

1. From $\Omega_{i}, i=1, \ldots, p$ choose $\Omega_{i^{\prime}}$ to be partitioned such that $\Omega_{i^{\prime}}$ satisfies the conditions of Proposition 1, i.e., it is splittable by Algorithm 3 .

2. Renumber $\Omega_{i}$ and $\mathbf{A}_{i}$, so that $\Omega_{i^{\prime}}, \mathbf{A}_{i^{\prime}}$ and $\mathbf{B}_{i^{\prime}}$ become $\Omega_{p}, \mathbf{A}_{p}$ and $\mathbf{B}_{p}$ respectively.

3. Set (with some abuse of notation) $\Omega=\Omega_{p}$. Find $\Omega_{1}, \Omega_{2}$ and $\Gamma$ satisfying the conditions of Proposition 1.

4. Set $\mathbf{A}=\mathbf{A}_{p}$ and apply Algorithm 3 and A.1 to obtain $\mathbf{A}^{1}, \mathbf{A}^{2}$ and $\mathbf{B}^{1}, \mathbf{B}^{2}$ respectively.

5. Set $\Omega_{p}=\Omega_{1}, \Omega_{p+1}=\Omega_{2}, \mathbf{A}_{p}=\mathbf{A}^{1}, \mathbf{A}_{p+1}=\mathbf{A}^{2}, \mathbf{B}_{p}=\mathbf{B}^{1}, \mathbf{B}_{p+1}=\mathbf{B}^{2}$.

The partitioning is repeated until the subdomain size is reduced to a desirable level, or until $\mathbf{A}_{i}$ become full. We assume that after $N_{c}-1$ steps of Algorithm 4 we partition A into submatrices $\mathbf{A}_{i} \in \mathbb{R}^{N_{i} \times N_{i}}, i=1, \ldots, N_{c}$ satisfying 2.4). Note that Proposition 2 can be recursively extended to $\mathbf{A}_{i}, i=1, \ldots, N_{c}$.

\section{Appendix B. Corner set removal algorithm.}


Let node $k$ belong to the "corner" set. Then for each $\Gamma_{i j}$ such that $k \in \Gamma_{i j}$ we introduce a hanging node $k_{i j}$ by copying $k$ and all elements $\left(\mathbf{P}_{i} \mathbf{A}_{i} \mathbf{P}_{i}^{T}+\mathbf{P}_{j} \mathbf{A}_{j} \mathbf{P}_{j}^{T}\right)_{k l}$ and $\left(\mathbf{P}_{i} \mathbf{B}_{i} \mathbf{P}_{i}^{T}+\mathbf{P}_{j} \mathbf{B}_{j} \mathbf{P}_{j}^{T}\right)_{k l}$. In particular, we define the elements of the modified matrix $\mathbf{A}^{\prime}$ as well as diagonal matrix $\mathbf{B}^{\prime}$ as

$$
\left(\mathbf{A}^{\prime}\right)_{k_{i j} l}=\left(\mathbf{A}^{\prime}\right)_{l k_{i j}}=\left(\mathbf{P}_{i} \mathbf{A}_{i} \mathbf{P}_{i}^{T}+\mathbf{P}_{j} \mathbf{A}_{j} \mathbf{P}_{j}^{T}\right)_{k l}
$$

and

$$
\left(\mathbf{B}^{\prime}\right)_{k_{i j} k_{i j}}=\left(\mathbf{P}_{i} \mathbf{B}_{i} \mathbf{P}_{i}^{T}+\mathbf{P}_{j} \mathbf{B}_{j} \mathbf{P}_{j}^{T}\right)_{k k} .
$$

Let $\mathcal{H}(k)$ be a set of all the constructed nodes with parent node $k$. Once the process is finished for all $\Gamma_{i j}$ containing node $k$, we remove node $k$ and all corresponding elements of $\mathbf{A}$ and $\mathbf{B}$.

Appendix C. Computation of matrix S-fraction coefficients via blockLanczos algorithm.

Matrix S-fraction coefficients $\mathbf{L}^{k}$ and $\widehat{\mathbf{L}}^{k}$ can be computed by applying a block version of Lanczos iteration. We use Lanczos approach because it allows to implement the well developed techniques of a standard block-Lanczos algorithm [30] to the matrix pencil $\left(\mathbf{A}^{m}, \mathbf{B}^{m}\right)$ to obtain a unitary $\mathbf{Q} \in \mathbb{R}^{\widetilde{K} m \times \widetilde{K} m}$ such that

$$
\widetilde{\mathbf{F}}^{m}\left(\omega^{2}\right)=\mathbf{R}^{T}\left(\mathbf{T}+\omega^{2} \mathbf{I}\right)^{-1} \mathbf{R}
$$

with

$$
\mathbf{T}=\mathbf{Q}^{T} \mathbf{A}^{m} \mathbf{Q}, \quad \mathbf{R}=\mathbf{Q}^{T} \mathbf{S}^{m}=\left[\beta_{1}, 0,0, \ldots, 0\right]^{T} .
$$

Here $\mathbf{T}$ is a Hermitian block tridiagonal matix

$$
\mathbf{T}=\left[\begin{array}{cccccc}
\alpha_{1} & \beta_{2} & 0 & 0 & \ldots & 0 \\
\beta_{2} & \alpha_{2} & \beta_{3} & 0 & \ldots & 0 \\
\vdots & \vdots & \vdots & \vdots & \ddots & \vdots \\
0 & \ldots & 0 & \beta_{m-1} & \alpha_{m-1} & \beta_{m} \\
0 & \ldots & 0 & 0 & \beta_{m} & \alpha_{m}
\end{array}\right]
$$

with blocks $\alpha_{k}=\alpha_{k}^{T} \in \mathbb{R}^{\widetilde{K} \times \widetilde{K}}$ and $\beta_{k}=\beta_{k}^{T} \in \mathbb{R}^{\widetilde{K} \times \widetilde{K}}, k=1,2, \ldots, m$.

The transfer function (C.1) can be expressed as

$$
\widetilde{\mathbf{F}}^{m}=\beta_{1} \mathbf{W}_{1},
$$

where matrices $\mathbf{W}_{k} \in \mathbb{R}^{\widetilde{K} \times \widetilde{K}}, k=1,2, \ldots, m+1$ satisfy the three-term relations that make apparent the connection to finite-difference schemes:

$$
\begin{aligned}
\alpha_{1} \mathbf{W}_{1}+\beta_{2} \mathbf{W}_{2}+\omega^{2} \mathbf{W}_{1} & =\beta_{1}, \\
\beta_{k} \mathbf{W}_{k-1}+\alpha_{k} \mathbf{W}_{k}+\beta_{k+1} \mathbf{W}_{k+1}+\omega^{2} \mathbf{W}_{k} & =\mathbf{0},
\end{aligned}
$$

with $\mathbf{W}_{m+1}=0$.

A second change of coordinates can simplify (C.3) even further. Specifically, we can transform C.4 to (5.3). The corresponding transformation is block-diagonal and 
is performed recursively for $k=1,2, \ldots, m$ using

$$
\begin{aligned}
\widehat{\mathbf{L}}^{k} & =\mathbf{G}_{k} \mathbf{G}_{k}^{T} \\
\mathbf{L}^{k} & =-\mathbf{G}_{k}^{T} \alpha_{k} \mathbf{G}_{k}{ }^{-1}-\mathbf{L}^{k-1}, \\
\mathbf{U}^{k} & =\mathbf{G}_{k} \mathbf{W}_{k}, \\
\mathbf{G}_{k+1} & =\left[\mathbf{G}_{k}^{T} \mathbf{L}^{k}\right]^{-1} \beta_{k+1},
\end{aligned}
$$

starting with $\mathbf{G}_{1}=\beta_{1}, \mathbf{L}^{0}=0$. After this transformation the transfer function becomes

$$
\widetilde{\mathbf{F}}^{m}\left(\omega^{2}\right)=\mathbf{U}^{1}=\mathbf{E}_{1}^{T}\left(\mathbf{L}+\omega^{2} \mathbf{I}\right) \mathbf{E}_{1} \widehat{\mathbf{L}}^{1}
$$

where $\mathbf{E}_{1}=[\mathbf{I}, 0,0, \ldots, 0]^{T} \in \mathbb{R}^{\widetilde{K} m \times \widetilde{K}}$ and $\mathbf{L}$ is the block-tridiagonal difference operator defined by $5.2-5.3$.

\section{Appendix D. Correction for corner set nodes.}

For any $i \neq j$ the node-wise MSSFROM solution $\widetilde{u}$ at the boundary nodes of $\Gamma_{i j}$ can be obtained as $\mathbf{S}_{i j} \widetilde{u}_{i j}$. Indeed, by construction of the modified graph without the corner set, we note that $\sum_{l \in \mathcal{H}(k)} \widetilde{u}_{l, t t} b_{l l}$ must approximate the fine-scale balance equation at the corner set node $k$, i.e. the term $u_{k, t t} b_{k k}$. Hence, assuming $\widetilde{u}_{l}$ is accurate at previous and current time step, we end up with the following correction procedure:

1. For all $k \in \Gamma^{\cap}$ compute MSSFROM solutions $\widehat{u} \in \mathbb{R}^{K_{h}}$ at hanging nodes $l \in \mathcal{H}(k)$ (we denote by $K_{h}$ the total number of hanging nodes):

$$
\widehat{u}_{l}=e_{l}^{T} \mathbf{S}_{i j} \widetilde{u}_{i j},
$$

where $l \in \Gamma_{i j}$ and $e_{l} \in \mathbb{R}^{K_{i j}}$ is the unit vector with component 1 at node $l$.

2. For all $k \in \Gamma^{\cap}$ define MSSFROM solution at the corner set node $k$ as $\frac{1}{b_{k k}} \sum_{l^{\prime} \in \mathcal{H}(k)} \widehat{u}_{l^{\prime}} b_{l^{\prime} l^{\prime}}$ and then compute the correction term $\delta \widehat{u} \in \mathbb{R}^{K_{h}}$ at hanging nodes $l \in \mathcal{H}(k)$ as

$$
\delta \widehat{u}_{l}=\frac{1}{b_{k k}} \sum_{l^{\prime} \in \mathcal{H}(k)} \hat{u}_{l^{\prime}} b_{l^{\prime} l^{\prime}}-\widehat{u}_{l} .
$$

3. Update $\widetilde{u}_{i j}$ by adding the correction term

$$
\delta \widetilde{u}_{i j}=\mathbf{S}_{i j}^{T} \mathbf{P}_{i j}^{H^{T}} \delta \widehat{u},
$$

where $\mathbf{P}_{i j}^{H} \in \mathbb{R}^{K_{h} \times K_{i j}}$ is the prolongation operator from $\Gamma_{i j}$ to hanging nodes $\cup_{k \in \Gamma \cap \mathcal{H}}(k)$ :

$$
\left(\mathbf{P}_{i j}^{H} x\right)_{k}=\left\{\begin{array}{ll}
x_{k}, & \text { if } k \in \Gamma_{i j} \\
0, & \text { otherwise }
\end{array} \quad, \quad \text { for } x \in \mathbb{R}^{K_{i j}}\right.
$$


[1] Yuri Arlinskii, Sergey Belyi, and Eduard Tsekanovskii. Conservative realizations of HerglotzNevanlinna functions, volume 217. Springer Science \& Business Media, 2011.

[2] Sergey Asvadurov, Vladimir Druskin, and Leonid Knizhnerman. Application of the difference gaussian rules to solution of hyperbolic problems. Journal of Computational Physics, 158(1):116-135, 2000.

[3] I. Babuska, G. Caloz, and J. Osborn. Special finite element methods for a class of second order elliptic problems with rough coefficients. SIAM J. Numer. Anal., 31:945-981, 1994.

[4] V Bolotnikov and L Sakhnovich. On an operator approach to interpolation problems for stieltjes functions. Integral Equations and Operator Theory, 35(4):423-470, 1999.

[5] Frank M. Callier and Charles A. Desoer. Linear System Theory, chapter Realization Theory, pages 295-314. Springer New York, New York, NY, 1991.

[6] Edoardo Di Napoli, Eric Polizzi, and Yousef Saad. Efficient estimation of eigenvalue counts in an interval. Numerical Linear Algebra with Applications, 2016.

[7] Vladimir Druskin and Leonid Knizhnerman. Gaussian spectral rules for second order finitedifference schemes. Numerical Algorithms, 25(1-4):139-159, 2000.

[8] Vladimir Druskin, Leonid Knizhnerman, Yousef Saad, and Mikhail Zaslavsky. On constructing the optimal basis for multi-scale spectral element methods via reduced order gramians. In preparation, 2016.

[9] Vladimir Druskin, Alexander Mamonov, Andrew E Thaler, and Mikhail Zaslavsky. Direct, nonlinear inversion algorithm for hyperbolic problems via projection-based model reduction. SIAM Journal on Imaging Science, 9(2):684-747, 2016.

[10] Vladimir Druskin, Alexander Mamonov, and Mikhail Zaslavsky. Multiscale mimetic reducedorder models for spectrally accurate wavefield simulations. SEG Technical Program Expanded Abstracts 2015, pages 3710-3715, 2015.

[11] Vladimir Druskin, Alexander Mamonov, and Mikhail Zaslavsky. S-fraction multiscale finitevolume method for spectrally accurate wavefield simulations. 77th EAGE Conference and Exhibition 2015, 2015.

[12] Vladimir Druskin and Shari Moskow. Three-point finite-difference schemes, padé and the spectral galerkin method. i. one-sided impedance approximation. Mathematics of computation, 71(239):995-1019, 2002.

[13] Yu M Dyukarev. Indeterminacy criteria for the stieltjes matrix moment problem. Mathematical Notes, 75(1-2):66-82, 2004.

[14] Y. Efendiev, J. Galvis, and T. Hou. Generalized multiscale finite element methods (gmsfem). Journal of Computational Physics, 251:116-135, 2013.

[15] Y. Efendiev and X.-H. Wu. Multiscale finite element methods for the problems with highly oscillatory coefficients. Numerische Math., 90(3):459-486, 2002.

[16] R.P. Fedorenko. Introduction to computational physics. Izdat. MPhTI (in Russian), 1994.

[17] Bernd Fritzsche, Bernd Kirstein, and Conrad Mädler. On matrix-valued stieltjes functions with an emphasis on particular subclasses. arXiv preprint arXiv:1506.01600, 2015.

[18] K. Gao, E. Chung, R. Gibson, and Y. Efendiev. Generalized multiscale finite-element method (gmsfem) for elastic wave propagation in heterogeneous, anisotropic media. submitted, http://arxiv.org/abs/1409.3550, 2014.

[19] W. Gawronski and J.-N. Juang. Model reduction in limited time and frequency intervals. International Journal of Systems Science, 21(2):349-376, 1990.

[20] Serkan Gugercin and Athanasios C Antoulas. A survey of model reduction by balanced truncation and some new results. International Journal of Control, 77(8):748-766, 2004.

[21] Stefan Guttel, Eric Polizzi, Ping Tak Peter Tang, and Gautier Viaud. Zolotarev quadrature rules and load balancing for the feast eigensolver. SIAM Journal on Scientific Computing, 37(4):A2100-A2122, 2015

[22] T.Y. Hou and X.-H Wu. A multiscale finite element method for elliptic problems in composite materials and porous media. Journal of Computational Physics, 134(1):169-189, 1997.

[23] P. Jenny, S. H. Lee, and H. Tchelepi. Multi-scale finite volume method for elliptic problems in subsurface flow simulation. Journal of Computational Physics, 187:47-67, 2003.

[24] IS Kac and MG Krein. On the spectral functions of the string. Amer. Math. Soc. Transl, 103(2):19-102, 1974.

[25] R. LeVeque and C. Zhang. Immersed interface methods for wave equations with discontinuous coefficients. Wave Motion, 25:237-263, 1997.

[26] Steffen Marburg. Computational Acoustics of Noise Propagation in Fluids - Finite and Boundary Element Methods, chapter Discretization Requirements: How many Elements per Wavelength are Necessary?, pages 309-332. Springer Berlin Heidelberg, Berlin, Heidelberg, 2008 .

[27] Vadim A Markel and John C Schotland. Homogenization of maxwell's equations in periodic 
composites: Boundary effects and dispersion relations. Physical Review E, 85(6):066603, 2012.

[28] George L Matthaei, Leo Young, and EMT Jones. Microwave filters, impedance-matching networks, and coupling structures, volume 1. Artech house, 1964.

[29] B. McMillan. Introduction to formal realizability theory. Bell System Tech. J, 31:442-464, 1952.

[30] Peter L. Montgomery. Advances in Cryptology - EUROCRYPT '95: International Conference on the Theory and Application of Cryptographic Techniques Saint-Malo, France, May 2125, 1995 Proceedings, chapter A Block Lanczos Algorithm for Finding Dependencies over GF(2), pages 106-120. Springer Berlin Heidelberg, Berlin, Heidelberg, 1995.

[31] S. Moskow, V. Druskin, T. Habashy, P. Lee, and S. Davydycheva. A finite difference scheme for elliptic equations with rough coefficients using a cartesian grid nonconforming to interfaces. Journal of Numerical Analysis, 36:442-464, 1999.

[32] Yuji Nakatsukasa and Roland W Freund. Computing fundamental matrix decompositions accurately via the matrix sign function in two iterations: The power of zolotarev's functions. SIAM Review, 58(3):461-493, 2016.

[33] Axel Ruhe. The rational krylov algorithm for nonsymmetric eigenvalue problems. iii: Complex shifts for real matrices. BIT Numerical Mathematics, 34(1):165-176, 1994.

[34] M. Zaslavsky and A. Pergament. Averaging algorithms and the support-operator method in elliptic problems with discontinuous coefficients. Journal of Computational Mathematics and Mathematical Physics, 45(9):1538-1548, 2005. 